\title{
Formation of an Accretion Flow
}

\author{
C. Bonnerot • N.C. Stone
}

Received: date / Accepted: date

\begin{abstract}
After a star has been tidally disrupted by a black hole, the debris forms an elongated stream. We start by studying the evolution of this gas before its bound part returns to the original stellar pericenter. While the axial motion is entirely ballistic, the transverse directions of the stream are usually thinner due to the confining effects of self-gravity. This basic picture may also be influenced by additional physical effects such as clump formation, hydrogen recombination, magnetic fields and the interaction with the ambient medium. We then examine the fate of this stream when it comes back to the vicinity of the black hole to form an accretion flow. Despite recent progress, the hydrodynamics of this phase remains uncertain due to computational limitations that have so far prevented us from performing a fully self-consistent simulation. Most of the initial energy dissipation appears to be provided by a self-crossing shock that results from an intersection of the stream with itself. The debris evolution during this collision depends on relativistic apsidal precession, expansion of the stream from pericenter, and nodal precession induced by the black hole spin. Although the combined influence of these effects is not fully understood, current works suggest that this interaction is typically too weak to significantly circularize the trajectories, with its main consequence being an expansion of the shocked gas. Global simulations of disc formation performed for simplified initial conditions find that the debris experiences additional collisions that cause its orbits to become more circular until eventually settling

C. Bonnerot

TAPIR, Mailcode 350-17

California Institute of Technology

Pasadena, CA 91125 (USA)

E-mail: bonnerot@tapir.caltech.edu

N.C. Stone

Racah Institute of Physics

The Hebrew University

Jerusalem, 91904 (Israel)

E-mail: nicholas.stone@mail.huji.ac.il
\end{abstract}


into a thick and extended structure. These works suggest that this process completes faster for more relativistic encounters due to the stronger shocks involved. It is instead significantly delayed if weaker shocks take place, allowing the gas to retain large eccentricities during multiple orbits. Radiation produced as the matter gets heated by circularizing shocks may leave the system through photon diffusion and participate in the emerging luminosity. This current picture of accretion flow formation drastically differs from that assumed in early analytical works. However, important aspects still remain to be understood at the time of writing, due to numerical challenges and the complexity of this process.

\section{Introduction}

In this chapter, we focus on the evolution of the debris around the black hole following stellar disruption, and most importantly the formation of an accretion flow from this matter. These two stages are described below in largely independent sections so that the reader is able to go through them in any order. While our understanding of isolated stream evolution is relatively robust, the later stage of accretion disc formation remains debated due to its greater complexity and associated numerical challenges. For the latter, we therefore present the major advances made so far while emphasizing the uncertainties of these current works.

Following the disruption of the star on its original parabolic trajectory, the debris evolves into an elongated stream due to the spread in orbital energy imparted to the stellar matter by the encounter with the black hole. While half of this gas is unbound and escapes the gravitational attraction of the compact object on hyperbolic orbits, the rest becomes bound and comes back to the stellar pericenter on highly eccentric trajectories. The hydrodynamics at play during this revolution of the stellar debris around the black hole is well understood thanks to the early work of Kochanek (1994), more recently revised by Coughlin et al. (2016a). During this phase, the stream gets stretched along its longitudinal direction due to the ballistic in-plane motion of the gas elements that evolve like test particles on a wide range of different elliptical orbits. In the other two transverse directions, the gas motion is usually specified by selfgravity that imposes a thin width such that the stream has a locally cylindrical geometry. The influence of various additional physical ingredients on this basic picture has been explored, including stream fragmentation into individual self-gravitating clumps, the recombination of hydrogen as the stream cools, the magnetic field inherited from the star and the interaction of the debris with the ambient gaseous medium. It is nevertheless fair to say that the basic picture of stream evolution described above is usually not strongly affected by this additional physics.

After the bound part of the dynamically cold stream has completed an entire orbit, it comes back near the black hole where it can start forming an accretion flow. As already mentioned, our understanding of this phase of evolu- 
tion is less secure than the earlier one due to the more complicated mechanisms involved and the numerical challenges of studying them. The returning stream is strongly compressed at pericenter, causing a nozzle shock whose main effect is to make the gas expand. Initial dissipation is provided by a collision of the stream with itself. The parameters of this collision are specified by the combination of relativistic apsidal precession, expansion from the nozzle shock, and nodal precession due to black hole spin. Local simulations of this interaction (e.g. Jiang et al. 2016b; Lu and Bonnerot 2019) find that the ensuing self-crossing shock dissipates part of the debris kinetic energy and results in an expansion of the gas distribution around the intersection point. However, performing a global study of the disc formation process is very computationally expensive and current investigations therefore rely on simplified initial conditions to circumvent this numerical burden. These numerical works (e.g. Hayasaki et al. 2013; Bonnerot et al. 2016; Shiokawa et al. 2015; Bonnerot and Lu 2019) find that the shocked gas experiences additional dissipation that causes the debris to move to more circular orbits and to eventually settle into a thick and extended accretion disc. Importantly, this final state may take a long time to be reached, and the gas likely retains significant eccentricities even after this process is completed. We emphasize that this evolutionary path differs significantly from that of many early analytic works, beginning with the pioneering study of Rees (1988), who envisioned the rapid formation of a compact and axisymmetric disc. While the above qualitative description of disc formation is relatively robust, there is so far no clear consensus on the detailed hydrodynamics at play for astrophysically realistic initial conditions. Nevertheless, our understanding of this process has now reached a level sufficient to better identify the main sources of uncertainty and start developing strategies to overcome them.

This chapter is arranged as follows. We start in Section 2 by describing the evolution of the stream around the black hole before it comes back to pericenter. The basic gas dynamics is first presented followed by the impact of additional physical processes. In Section 3, we explain the current understanding of how the returning debris forms an accretion flow. We start by describing the initial sources of dissipation in a largely analytical fashion and then present the various numerical investigations of the disc formation process along with its main consequences. Finally, Section 4 contains a summary and our concluding remarks.

\section{Stream evolution prior to pericenter return}

\subsection{Basic stream trajectory and geometry}

The trajectory of the debris stream mostly results from the disruption process discussed in the Disruption Chapter, but we start by recalling the most important properties below. The star is disrupted by the tidal force of the black 
hole if its pericenter distance $R_{\mathrm{p}}$ is lower than the tidal radius

$$
R_{\mathrm{t}}=R_{\star}\left(\frac{M_{\mathrm{h}}}{M_{\star}}\right)^{1 / 3}=0.47 \mathrm{AU}\left(\frac{\mathrm{M}_{\mathrm{h}}}{10^{6} \mathrm{M}_{\odot}}\right)^{1 / 3},
$$

with the depth this encounter being characterized by the penetration factor $\beta=R_{\mathrm{t}} / R_{\mathrm{p}}$. Here, $M_{\mathrm{h}}$ denotes the mass of the compact object while $M_{\star}$ and $R_{\star}$ are the stellar mass and radius, respectively. As in the above equation, the numerical values in the remaining of the chapter are usually given assuming a solar-type star with $M_{\star}=\mathrm{M}_{\odot}$ and $R_{\star}=\mathrm{R}_{\odot}$ unless stated otherwise. The disruption imparts a spread in specific orbital energy (Rees 1988; Stone et al. 2013)

$$
\Delta \varepsilon=\frac{G M_{\mathrm{h}} R_{\star}}{R_{\mathrm{t}}^{2}},
$$

to the debris that makes half of it unbound while the rest gets bound to the black hole. The spread in angular momentum is however negligible (see for example the figure 2 from Cheng and Bogdanović 2014), which implies in particular that all the stream elements keep a pericenter distance equal to that of the original star. As the gas keeps orbiting the black hole, it is usually a good approximation to consider its trajectory along the orbital plane as perfectly ballistic. This implies that the gas evolves into an elongated stream with the unbound part escaping to large distances while the bound gas returns to the stellar pericenter after a finite time. In between, a fraction of the debris is marginally-bound, remaining on the parabolic trajectory that the star followed until its disruption. The gas located at the most bound extremity of the stream has a semi-major axis

$$
a_{\min }=\frac{R_{\star}}{2}\left(\frac{M_{\mathrm{h}}}{M_{\star}}\right)^{2 / 3}=23 \mathrm{AU}\left(\frac{\mathrm{M}_{\mathrm{h}}}{10^{6} \mathrm{M}_{\odot}}\right)^{2 / 3},
$$

making use of equation (2). It is the first to come back to the black hole following the disruption after a time equal to its orbital period given by

$$
t_{\min }=2^{-1 / 2} \pi\left(\frac{G M_{\star}}{R_{\star}^{3}}\right)^{-1 / 2}\left(\frac{M_{\mathrm{h}}}{M_{\star}}\right)^{1 / 2}=41 \mathrm{~d}\left(\frac{\mathrm{M}_{\mathrm{h}}}{10^{6} \mathrm{M}_{\odot}}\right)^{1 / 2},
$$

according to Kepler's third law and equation (3). This stream element is highly-eccentric with an eccentricity $e_{\min }=1-R_{\mathrm{p}} / a_{\min }$ given by

$$
1-e_{\min }=\frac{2}{\beta}\left(\frac{M_{\mathrm{h}}}{M_{\star}}\right)^{-1 / 3}=0.02 \beta\left(\frac{M_{\mathrm{h}}}{10^{6} \mathrm{M}_{\odot}}\right)^{-1 / 3}
$$

using equations (1) and (3). The mass fallback rate $\dot{M}_{\mathrm{fb}}$ at which the stellar matter comes back to the black hole is specified by how orbital energy is distributed among the stream. Assuming a flat distribution yields $\dot{M}_{\mathrm{fb}}=$ $\dot{M}_{\mathrm{p}}\left(t / t_{\text {min }}\right)^{-5 / 3}$ with a peak value of

$$
\dot{M}_{\mathrm{p}}=\frac{M_{\star}}{3 t_{\min }}=3 \mathrm{M}_{\odot} \mathrm{yr}^{-1}\left(\frac{\mathrm{M}_{\mathrm{h}}}{10^{6} \mathrm{M}_{\odot}}\right)^{-1 / 2}
$$


such that the integrated amount of returning mass is that $M_{\star} / 2$ of the bound debris.

The geometry of the stream is well approximated by a cylinder whose thickness and elongation evolve in time. The latter is entirely determined by the ballistic motion of the gas along the orbital plane. In particular, one can specify the dependence of the length $l(R)$ of a stream element of fixed mass on its distance $R$ from the black hole. At early times $t \lesssim t_{\min }$ when most of the gas still follows a near-parabolic orbit, this length scales as (Coughlin et al. 2016a)

$$
l \propto R^{2}
$$

This result was derived by solving the equations of gas dynamics for the marginally-bound part of the stream but it can also be recovered in the following more intuitive way. Immediately after the disruption, the stream is still close to the tidal radius with a size similar to that of the original star, which implies $l\left(R_{\mathrm{t}}\right) \approx R_{\star}$. As time goes on, the gas distribution stretches and some debris falls back in the vicinity of the black hole after a complete revolution around it. The stream has therefore a length similar to its mean distance from the compact object that is of order the semi-major axis of the most bound debris, resulting in $l\left(a_{\min }\right) \approx a_{\min }$. These two estimates of the stream length can be combined to prove the quadratic dependence of the length with distance since $l\left(a_{\min }\right) / l\left(R_{\mathrm{t}}\right) \approx\left(a_{\min } / R_{\mathrm{t}}\right)^{2}$ using equations (1) and (3). The evolution of the stream length can also be expressed in terms of the time $t$ since disruption as $l \propto t^{4 / 3}$ making use of the dependence $R \propto t^{2 / 3}$ valid for a near-parabolic orbit. After a time $t \gtrsim t_{\text {min }}$, the trajectories of most gas elements start to significantly deviate from a parabolic orbit and the above scaling becomes invalid. Once they have escaped the gravity of the black hole, the unbound stream elements move at a constant speed such that their distance from the compact object evolves as $R \propto t$. Because the marginally-bound gas reaches a velocity close to zero, the length of this part of the stream follows the same scaling with $l \propto R \propto t$ (Coughlin et al. 2016a).

We now focus on the thickness of the gas distribution. Similarly to the above calculation, it is possible to determine the evolution of the width $H$ of a stream element containing a fixed amount of mass as it orbits the black hole. While the densest stream elements have their transverse motion specified by hydrostatic equilibrium between self-gravity and gas pressure, the ones with lower densities have their width set by the shear induced by the tidal force from the black hole. The critical density separating these two regimes can be found by comparing the specific tidal and self-gravity forces acting on the stream that are given by $f_{\text {tid }} \approx G M_{\mathrm{h}} H / R^{3}$ and $f_{\mathrm{sg}} \approx G m /(H l) \approx G \rho H$, respectively. To obtain the latter expression, the Gauss's theorem has been used as well as the relation $m \approx \rho H^{2} l$ between the mass $m$ and density $\rho$ of a stream element that exploits its cylindrical geometry. Therefore, an element has its width set by hydrostatic equilibrium with $f_{\mathrm{sg}}>f_{\text {tid }}$ if $\rho>\rho_{\mathrm{c}} \equiv M_{\mathrm{h}} / R^{3}$ where $\rho_{\mathrm{c}}$ denotes the critical density. The next step is to determine the scaling that the stream width obeys in the self-gravity and shear-dominated regimes corresponding to 


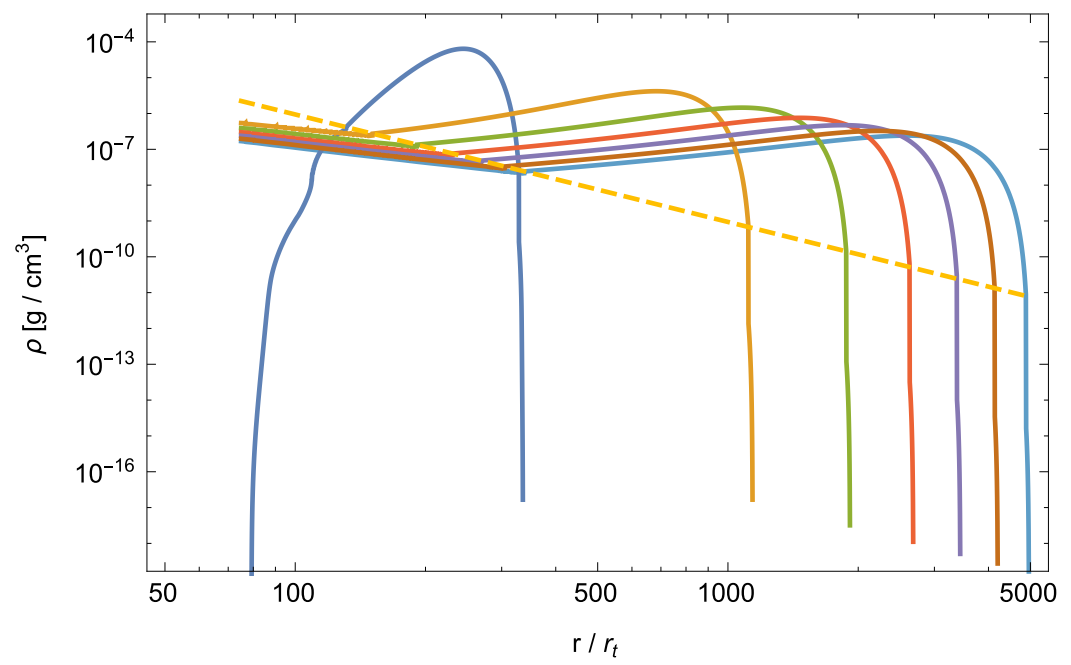

Fig. 1 Stream density as function of distance from the black hole at different times starting from $t=32 \mathrm{~d}$ (leftmost, dark blue line) and ending at $t=620 \mathrm{~d}$ (rightmost, light blue line) with an interval of $98 \mathrm{~d}$ between them (Coughlin et al. 2016a). The star has a solar mass and radius and is disrupted by a black hole of mass $M_{\mathrm{h}}=10^{6} \mathrm{M}_{\odot}$ with a penetration factor of $\beta=1$. The gas evolution is assumed to be adiabatic with $\gamma=5 / 3$. The yellow dashed line shows the critical density $\rho_{\mathrm{c}}=M_{\mathrm{h}} / R^{3}$ that separates between the self-gravity and shear-dominated regimes.

$\rho>\rho_{\mathrm{c}}$ and $\rho<\rho_{\mathrm{c}}$, respectively. When the tidal force dominates, the width of the stream evolves according to the homologous scaling $H \propto R$. This is because the gas parcels on its cylindrical surface experience a gravitational attraction directed in the radial direction that causes a compression of the stream element as it moves inwards. Instead, hydrostatic equilibrium imposes that the selfgravity force $f_{\mathrm{sg}} \approx G \rho H$ follows the gas pressure force $f_{\text {gas }}=\nabla P / \rho \approx P /(\rho H)$, where $P$ denotes the gas pressure that we assume to evolve according to the polytropic relation $P \propto \rho^{\gamma}$. Imposing $f_{\text {gas }} \propto f_{\text {sg }}$ then leads after some algebra to the relation $H \propto l^{(2-\gamma) / 2(\gamma-1)}$. When $t \lesssim t_{\text {min }}$, the stream length follows equation (7), which yields

$$
H \propto R^{\frac{2-\gamma}{\gamma-1}} .
$$

Assuming an adiabatic evolution with $\gamma=5 / 3$, the width scales as $H \propto$ $R^{1 / 2} \propto t^{1 / 3}$ that corresponds to a density evolution as $\rho \propto\left(H^{2} l\right)^{-1} \propto R^{-3} \propto$ $t^{-2}$. The unbound elements follow $l \propto R$ at late times $t \gtrsim t_{\text {min }}$ such that $H \propto R^{(2-\gamma) / 2(\gamma-1)} \propto R^{1 / 4} \propto t^{1 / 4}$. This latter scaling was found first by Kochanek (1994) who assumed the relation $l \propto R$ in the stream. It was later revised to $H \propto R^{1 / 2}$ at early times by Coughlin et al. (2016a) that makes use of the correct length evolution. Importantly, these scalings show that self-gravity confines the width of the stream such that it evolves slower with distance from the black hole than for the homologous scaling imposed by the tidal force.

For grazing encounters with $\beta \approx 1$, the stellar disruption results in a large fraction of the stream being dominated by self-gravity. This can for exam- 


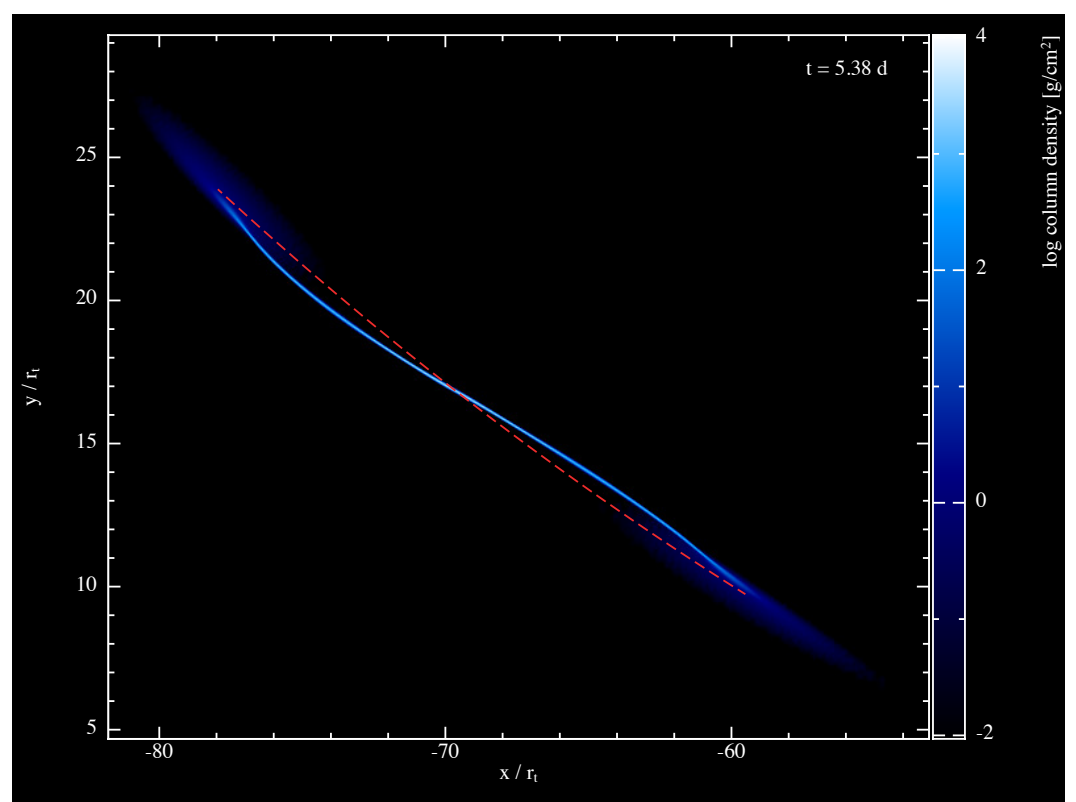

Fig. 2 Column density of the stream of debris a time of $t=5.38 \mathrm{~d}$ after the disruption of a star of solar mass and radius by a black hole of mass $M_{\mathrm{h}}=10^{6} \mathrm{M}_{\odot}$ with a penetration factor of $\beta=1$ (Coughlin and Nixon 2015).

ple be seen from Fig. 1 that shows stream density profiles at different times with solid lines of different colours according to the semi-analytical model of Coughlin et al. (2016a). It considers the disruption of a solar-type star by a black hole of mass $M_{\mathrm{h}}=10^{6} \mathrm{M}_{\odot}$ with a penetration factor $\beta=1$, additionally assuming an adiabatic gas evolution with $\gamma=5 / 3$. Most of the stream elements have a density above the dashed yellow line representing the critical value $\rho_{\mathrm{c}}=M_{\mathrm{h}} / R^{3}$, meaning that they are in hydrostatic equilibrium. This implies that the majority of the gas distribution is able to remain thin thanks to the confinement by self-gravity with a typical width given at $R \approx a_{\text {min }}$ by $H / R_{\star} \approx\left(a_{\min } / R_{\mathrm{t}}\right)^{1 / 2} \approx\left(M_{\mathrm{h}} / M_{\star}\right)^{1 / 6} \lesssim 10$ according to the scaling of equation (8). Shear-dominated elements are only present at the extremities of the stream due to the lower densities inherited from the original stellar density profile. ${ }^{1}$ Numerical investigations of the stream evolution by Guillochon et al. (2014) and Coughlin et al. (2016a) confirm these expectations. It can for example be seen from Fig. 2 that shows the column density inside the stream obtained from a simulation that adopts the same parameters as the above semi-analytical study. The largest densities are reached in the central parts where the gas distribution remains thin owing to its self-gravity. Instead, the

\footnotetext{
1 Remarkably, the width evolution becomes homologous with $H \propto R$ near the black hole due to the increased tidal force that corresponds to an up-turn in the density profile seen at $R \lesssim 200 R_{\mathrm{t}}$ in Fig. 1
} 
lower densities near the two ends of the stream imply that these regions are shear-dominated and therefore more extended.

Deep disruptions with $\beta \gtrsim 3$ result in heating since the star gets compressed during its disruption, as discussed in the Disruption Chapter. The ensuing expansion can cause most of the gas to have their densities decreased to $\rho<\rho_{\mathrm{c}}$. In this situation, one would expect that the stream is entirely sheardominated with a width that evolves homologously until late times (Kochanek 1994; Coughlin et al. 2016a). This scenario is favoured by the fact that the density evolves at early times as $\rho \propto\left(H^{2} l\right)^{-1} \propto R^{-4}$ in this regime such that the condition $\rho<\rho_{\mathrm{c}}=M_{\mathrm{h}} / R^{3}$ remains satisfied if it initially is. However, recent simulations by Steinberg et al. (2019) considering $\beta \geq 5$ find that a significant fraction of the stream is nevertheless able to recollapse under its self-gravity, possibly due to the presence of a weak caustic in the in-plane motion of the debris (Coughlin et al. 2016b). Although it contains more shear-dominated matter than for a grazing encounter, the resulting gas distribution remains therefore qualitatively similar to that described above for $\beta \approx 1$.

\subsection{Additional physics}

\subsubsection{Gravitational fragmentation}

As the stream expands, adiabatic cooling can trigger gravitational fragmentation with individual clumps forming within it. This phenomenon was first found in simulations by Coughlin and Nixon (2015) that consider a tidal disruption with $\beta=1$ assuming an adiabatic evolution with $\gamma=5 / 3$. Although the perturbation triggering fragmentation in this work has likely a numerical origin, a physical one has been identified by Coughlin et al. (2016b) that is produced by the in-plane compression of the star as it is being disrupted by the black hole. This compression is due to the fact that, when the star is at the pericenter of its trajectory, the gas that has already passed this location decelerates while that lagging behind keeps accelerating. This results in a density increase happening a few hours after the disruption followed by oscillations as the stream settles back to hydrostatic equilibrium. The associated perturbation was proposed to destabilize the stream and trigger gravitational fragmentation but additional mechanisms likely exist such as the interaction with the surrounding medium discussed in Section 2.2.4.

The conditions for gravitational fragmentation have been examined more precisely by Coughlin et al. (2016a). It requires that the free-fall time $t_{\mathrm{ff}} \approx$ $(\rho G)^{-1 / 2}$ is locally shorter than the dynamical time $t_{\mathrm{dyn}} \approx\left(G M_{\mathrm{h}} / R^{3}\right)^{-1 / 2}$ necessary for the stream to get stretched in the radial direction. The condition $t_{\text {ff }}<t_{\text {dyn }}$ then translates to $\rho \gtrsim M_{\mathrm{h}} / R^{3}=\rho_{\mathrm{c}}$, which is identical to that derived in Section 2.1 for a stream element to be in hydrostatic equilibrium. In this regime, the density scales as $\rho \propto R^{-2 /(\gamma-1)}$, implying that the condition for fragmentation remains valid at later times as long as $\gamma \geq 5 / 3$. This analysis suggests that clump formation is only possible for adiabatic exponents 
larger than this critical value and occurs faster when $\gamma$ is increased. Numerical simulations by Coughlin et al. (2016b) confirm this trend by studying the stream evolution for different values of the adiabatic exponent. In reality, $\gamma>5 / 3$ requires that the stream cools non-adiabatically, which can for instance be achieved through recombination when the gas becomes optically thin (see Section 2.2.2).

The fate of these clumps depends on whether they belong to the bound or unbound part of the stream. The bound ones affect the density of the gas falling back near the black hole with the first one reaching the compact object about two years after disruption. Accretion of these discrete fragments onto the black hole could lead to a variable emission (Coughlin and Nixon 2015). Instead, the unbound clumps escape the black hole, which constitutes a new kind of high-velocity objects (Coughlin et al. 2016b). The distribution of these fragments has been studied more in detail by Girma and Guillochon (2018) who find that $\sim 10^{7}$ of them have been launched from $\mathrm{Sgr} \mathrm{A}^{\star}$ within the life of our galaxy.

\subsubsection{Recombination}

Immediately after the disruption, the gas is fully ionized with a temperature of $T_{\star} \approx G M_{\star} m_{\mathrm{p}} /\left(k_{\mathrm{B}} R_{\star}\right)=2 \times 10^{7} \mathrm{~K}$, as shown in the Disruption Chapter. Here, $k_{\mathrm{B}}$ denotes the Boltzmann constant while $m_{\mathrm{p}}$ is the proton mass. As it subsequently evolves around the black hole, this debris adiabatically cools until it reaches a temperature $T_{\text {rec }} \approx 10^{4} \mathrm{~K}$, at which hydrogen recombines. This decrease in temperature starts when the stream has stretched by a significant amount, which requires a time $t_{\mathrm{str}} \approx\left(R_{\mathrm{t}}^{3} / G M_{\mathrm{h}}\right)^{1 / 2} \approx 1 \mathrm{~h} \mathrm{sim}$ ilar to the dynamical timescale near the tidal radius that is also the stellar dynamical timescale according to equation (1). As the stream expands, the temperature evolves as $T \propto \rho^{2 / 3} \propto t^{-4 / 3}$ using the scaling $\rho \propto t^{-2}$ valid at early times assuming hydrostatic equilibrium (Section 2.1 ), which leads to $T \approx T_{\star}\left(t / t_{\mathrm{str}}\right)^{-4 / 3}$. Setting $T=T_{\text {rec }}$ then implies that recombination sets in after a time $t_{\text {rec }} \approx t_{\text {str }}\left(T_{\star} / T_{\text {rec }}\right)^{3 / 4} \approx 10 \mathrm{~d}$ (Coughlin et al. 2016a).

The effect of recombination on the stream depends on its optical depth (Kochanek 1994; Coughlin et al. 2016a; Kasen and Ramirez-Ruiz 2010). By the time it occurs, the majority of the gas is still very optically thick, implying that this debris gets heated by the additional energy injection. During this process, the stream is imposed to evolve at a roughly constant temperature and becomes overpressured, causing an increase of its thickness and the end of its confinement by self-gravity. Since the stream outer layers are less dense, they are able to radiatively cool and become neutral that causes a decrease of the opacity since less free electrons are present. This process drives a transparency wave that propagates from the outer regions through the entire stream in a few days while producing an optical transient emission with a radiated luminosity $L_{\text {rec }} \lesssim 10^{41} \operatorname{erg~s}^{-1}$ (Kasen and Ramirez-Ruiz 2010). 


\subsubsection{Magnetic fields}

The magnetic field of the star gets transferred to the debris upon disruption and is subsequently carried by the stream in its evolution around the black hole. Its magnetic strength is then entirely specified by the stream geometry derived in Section 2.1 according to magnetic flux conservation. This condition imposes that the magnetic field parallel to the direction of stream elongation evolves as $B_{\|} \propto S_{\|}^{-1}$ where $S_{\|} \approx H^{2}$ denotes the surface the corresponding field lines go through. This results in $B_{\|} \propto R^{-1}$ according to equation (8) and assuming $\gamma=5 / 3$. Similarly, the magnetic field orthogonal to the direction of stream stretching evolves as $B_{\perp} \propto S_{\perp}^{-1}$ with $S_{\perp} \propto H l$ such that $B_{\perp} \propto R^{-5 / 2}$ additionally using equation (7). The fact that the parallel component decreases much slower than the perpendicular one implies that the field lines tend to align along the stream longitudinal direction.

Given this evolution, it is possible to evaluate the relative importance of magnetic and gas pressure inside the stream. Assuming that the field lines are all parallel to the direction of stream elongation, magnetic pressure follows $P_{\text {mag }} \approx B_{\|}^{2} \propto R^{-2}$ while the gas pressure scales as $P_{\text {gas }} \propto \rho^{5 / 3} \propto R^{-5}$ for an adiabatic evolution. This shows that the ratio of magnetic to gas pressure increases as $P_{\text {mag }} / P_{\text {gas }} \propto R^{3} \propto t^{2}$. Magnetic pressure takes over gas pressure with $P_{\mathrm{mag}} / P_{\mathrm{gas}}=1$ after a time given by $t_{\mathrm{mag}} \approx t_{\mathrm{str}} \beta_{\star}^{1 / 2}$ where $t_{\mathrm{str}} \approx 1 \mathrm{~h}$ has been defined in Section 2.2.2 while $\beta_{\star}$ denotes the initial plasma beta, defined as the ratio of gas to magnetic pressure inside the original star. This parameter can be evaluated as $\beta_{\star} \approx 10^{16}\left(B_{\star} / 1 G\right)^{-2}$ denoting by $B_{\star}$ the stellar magnetic field. Decreasing $t_{\mathrm{mag}}$ to about a month such that it becomes of order the orbital period $t_{\min }$ of the most bound debris defined in equation (4) therefore requires $B_{\star} \gtrsim 10^{5} G$ that corresponds to a highly-magnetized star. In this situation, the stream gets dominated by magnetic pressure that can cause a fast increase of its width and the end of confinement by self-gravity (Guillochon and McCourt 2017; Bonnerot et al. 2017).

\subsubsection{Stream-disc interaction}

So far, we have assumed that the stream evolves in isolation but the debris may in fact be affected by the ambient gaseous medium present around the black hole. A possible interaction relates to the Kelvin-Helmholtz instability that can develop at the interface between the debris and the surrounding matter, resulting in an efficient mixing of these two fluids. A given stream element is affected by this process if the instability has fully developed by the time the corresponding debris comes back to pericenter. Using this criterion, Bonnerot et al. (2016) finds that the entire stream can be impacted for the disruptions of red giants by black holes of mass $M_{\mathrm{h}} \gtrsim 10^{9} \mathrm{M}_{\odot}$. In this situation, most of the debris likely gets mixed with the ambient medium that can result in a sub-luminous event. Although this type of disruptions is promising to probe the high-end of the black hole mass function (MacLeod et al. 2014), the above process may significantly complicate their detection. If the galaxy contains a 
denser disc, tidal streams produced by main sequence stars can also be affected by the instability. This possibility has been put forward to explain the sudden drop in luminosity detected from some events (Kathirgamaraju et al. 2017) and could be involved in the capture of the disc magnetic field by the debris stream (Kelley et al. 2014).

Additional effects can take place if a TDE happens in an active galactic nucleus that contains a dense accretion flow. ${ }^{2}$ Using hydrodynamical simulations, Chan et al. (2019) studied the interaction of a debris stream with such a disc and find two different outcomes depending on the density of the incoming stellar matter. For low densities, the stream deposits most of its kinetic energy in the disc to get promptly absorbed by it. A stream of higher density can instead pierce through the disc while significantly perturbing the inflowing matter that can result in a fraction of the stream getting unbound and a depletion of the inner part of the accretion flow.

\section{Disc formation}

After a revolution around the black hole, the bound part of the stream comes back to the stellar pericenter. We are interested here in the fate of this gas and how it evolves to form an accretion flow. A simple although unrealistic scenario consists in the immediate formation of a circular disc from the infalling debris near the circularization radius $R_{\text {circ }} \approx 2 R_{\mathrm{p}}$ that corresponds to the lowest energy state for the gas if each fluid element conserves its specific angular momentum. ${ }^{3}$ In practice, this full circularization of the trajectories requires that a specific energy

$$
\Delta \varepsilon_{\mathrm{circ}} \approx \frac{G M_{\mathrm{h}}}{4 R_{\mathrm{p}}}
$$

is entirely lost from the system. ${ }^{4}$ The early analytical work by Rees (1988) proposes that the gas is actually able to rapidly dissipate this large amount of orbital energy such that disc formation happens in less than the fallback time given by equation (4). Furthermore, the outcome was predicted to consist of an axisymmetric accretion flow located near $R_{\text {circ }}$ that is thick due to the inability of photons to promptly radiate away the excess internal energy of the gas. As mentioned above, this picture is however not supported by current

\footnotetext{
2 Note that the rate of tidal disruptions in such systems may be increased due to the modification of the gravitational potential by the mass of the disc (Karas and Šubr 2007), as discussed in more details in the Rates Chapter.

3 If all the debris have the same specific angular momentum as that of the star on its original parabolic orbit, $l_{\star}=\sqrt{2 G M_{\mathrm{h}} R_{\mathrm{p}}}$, the lowest energy state is a circular orbit at a distance $R_{\text {circ }}=l_{\star}^{2} / G M_{\mathrm{h}}=2 R_{\mathrm{p}}$ from the black hole. Although the most bound part of the stream is on an elliptical orbit, this estimate remains valid since its typical eccentricity is $e_{\min }=0.98 \approx 1$ as shown in equation $(5)$.

4 Although the most bound part of the stream comes back to pericenter with an already negative energy given by equation (2), it is much larger than that required to reach complete circularization, since $\Delta \varepsilon / \Delta \varepsilon_{\text {circ }} \approx R_{\mathrm{p}} / a_{\min } \ll 1$, and therefore $\Delta \varepsilon$ is irrelevant in the computation of the energy loss necessary to reach this configuration.
} 
investigations. Recent studies find instead that the early interactions experienced by the returning stream typically dissipate an energy much lower than $\Delta \varepsilon_{\text {circ }}$, implying a slower disc formation that can require more than a fallback time to complete. The resulting gas distribution is also typically more extended due to the large internal energy retained such that a significant fraction of the debris can be present at distances much larger than the stellar pericenter. ${ }^{5}$ Additionally, we emphasize that the gas evolution is expected to be qualitatively different depending on the region of parameter space considered. Although we refer to the gas structure formed from the debris around the black hole as a "disc", it can significantly differ from the simplest disc models (e.g. Shakura and Sunyaev 1973) used in the literature. ${ }^{6}$ Despite recent progress in the understanding of disc formation, it is important to highlight that no clear consensus has so far been reached concerning the hydrodynamics of this process, largely due to numerical limitations that have so far prevented fully realistic global simulations of disc formation. In the following, we attempt to provide a description of the current status of theoretical knowledge, remaining as agnostic as possible while also highlighting (i) the main sources of uncertainties and (ii) the areas where secure conclusions appear possible.

In Section 3.1, we describe the dissipation mechanisms taking place shortly after the return of the stream near the black hole. The numerical challenges inherent to a global simulation of disc formation are discussed in Section 3.2 along with the compromises made so far to alleviate them. Section 3.3 presents the results of these numerical works that capture the evolution of the gas as the accretion flow assembles. The radiative efficiency of the different shocks involved in this process and the resulting emission are evaluated in Section 3.4. Finally, Section 3.5 describes the consequences of disc formation on the subsequent phase of accretion onto the black hole.

\subsection{Early sources of dissipation}

We start by discussing dissipation mechanisms taking place shortly after the debris comes back near the black hole, including the nozzle shock and the self-crossing shock. The nozzle shock is due to a strong compression of the stream during pericenter passage that results in an expansion of the gas as it then moves outward. Later on, an intersection of the stream with itself causes a self-crossing shock that can induce a significant modification of the gas trajectories.

\footnotetext{
5 There is also observational evidence supporting the notion that disc formation occurs differently from what early works assumed. For example, a compact accretion disc cannot reproduced the high level of optical emission detected from many events (Lodato and Price 2010; Miller 2015). Additionally, most TDEs have an integrated energy lower than $M_{\star} \Delta \varepsilon_{\text {circ }} \approx 10^{52} \mathrm{erg}$, by at least an order of magnitude. This "inverse energy crisis" is discussed in Section 3.5.2 along with possible solutions.

6 As will be described more precisely in Section 3.3, it is for example possible that this disc remains globally eccentric, or inclined with respect to the black hole spin.
} 


\subsubsection{Nozzle shock}

An early source of dissipation occurs during the passage of the stream at pericenter. In this region, the gas trajectories are initially specified by the gravity of the black hole. A consequence is that the fluid elements furthest from the original stellar orbital plane move on inclined orbits that intersect near pericenter, resulting in a compression of the stream in the vertical direction. This effect is analogous to the compression experienced by a star if it is disrupted on a plunging trajectory with $\beta>1$. Here, the stream is always inside its own tidal radius, with an effective penetration factor $\beta_{\mathrm{s}} \gg \beta$ since its density has decreased compared to the stellar value by several orders of magnitude (see Section 2). The compression results in a nozzle shock, during which the kinetic energy associated with vertical gas motion near pericenter gets dissipated.

As discussed in the Disruption Chapter, the vertical velocity associated to the stellar compression is given approximately by $v_{\mathrm{z}} \approx \alpha v_{\mathrm{p}} \approx \beta c_{\mathrm{s}, \star}$ where $c_{\mathrm{s}, \star}=\sqrt{G M_{\star} / R_{\star}}$ denotes the stellar sound speed. Here, $v_{\mathrm{p}}=\left(G M_{\mathrm{h}} / R_{\mathrm{p}}\right)^{1 / 2}$ is a characteristic velocity near pericenter while $\alpha=R_{\star} / \sqrt{R_{\mathrm{t}} R_{\mathrm{p}}}$ denotes the inclination angle between the orbital plane of the compressed gas and that of the stellar center of mass (Carter and Luminet 1982). As pointed out by Guillochon et al. (2014), this expression can be extrapolated to study the compression of the returning debris, for which the vertical velocity becomes $v_{\mathrm{z}, \mathrm{s}} \approx \beta_{\mathrm{s}} c_{\mathrm{s}, \mathrm{s}}$ where $c_{\mathrm{s}, \mathrm{s}}$ represents the sound speed inside the stream. Denoting by $\rho_{\star}$ and $\rho_{\mathrm{s}}$ the stellar and stream densities, the ratio of penetration factors is given by $\beta_{\mathrm{s}} / \beta \approx\left(\rho_{\star} / \rho_{\mathrm{s}}\right)^{1 / 3}$ since the tidal radius scales as $R_{\mathrm{t}} \propto \rho^{-1 / 3}$ while the pericenter distance is unchanged. Similarly, the sound speed ratio can be evaluated as $c_{\mathrm{s}, \mathrm{s}} / c_{\mathrm{S}, \star} \approx\left(\rho_{\star} / \rho_{\mathrm{S}}\right)^{-1 / 3}$ since $c_{\mathrm{S}} \propto(P / \rho)^{1 / 2} \propto \rho^{1 / 3}$ under the legitimate assumption of an adiabatic evolution for the debris with an adiabatic exponent $\gamma=5 / 3$. The density ratio therefore cancels out such that $v_{\mathrm{z}, \mathrm{s}} \approx v_{\mathrm{z}}{ }^{7}$

The nozzle shock dissipates a large fraction of the specific kinetic energy associated with the vertical motion of the stream,

$$
\Delta \varepsilon_{\mathrm{no}}=v_{\mathrm{z}, \mathrm{s}}^{2} \approx \beta^{2} \frac{G M_{\star}}{R_{\star}},
$$

where we have used the above estimates for the vertical speed of $v_{\mathrm{z}, \mathrm{s}}=$ $\beta \sqrt{G M_{\star} / R_{\star}}$. It follows that $\Delta \varepsilon_{\mathrm{no}} / \Delta \varepsilon_{\text {circ }} \approx \alpha^{2} \approx 10^{-4} \beta\left(M_{\mathrm{h}} / 10^{6} M_{\star}\right)^{-2 / 3}$, making use of equation (9). This calculation suggests that the dissipation provided by the nozzle shock is negligible compared to that necessary to completely circularize the orbits except for very low black hole masses $M_{\mathrm{h}} \lesssim$ $10^{4} \mathrm{M}_{\odot}$, where $\Delta \varepsilon_{\text {no }} \gtrsim 10^{-1} \Delta \varepsilon_{\text {circ }}$ (Guillochon et al. 2014). The internal energy injected in the shocked gas can nevertheless cause the stream to expand significantly after leaving pericenter, an effect captured by several sim-

7 This relation can also be understood from the fact that the stream has a width similar to the original stellar radius when it comes back near pericenter. Because the compression takes place near the tidal radius, it occurs on the dynamical timescale of the star, which leads to $v_{\mathrm{z}, \mathrm{s}} \approx \sqrt{G M_{\star} / R_{\star}}$. 
ulations described in Section 3.3, and especially strong for those considering an intermediate-mass black hole (Ramirez-Ruiz and Rosswog 2009; Shiokawa et al. 2015; Guillochon et al. 2014) or a large penetration factor (Sadowski et al. 2016). As discussed more in details in Section 3.1.2, this expansion can have important consequences on the later evolution of the gas.

So far, the nozzle shock has not been studied in a systematic fashion for typical parameters of the problem and our understanding of it is therefore limited. Important uncertainties concern the exact amount of dissipated energy and its consequences for the subsequent gas evolution. One possibility is that a fraction of the kinetic energy associated with the in-plane motion is dissipated during this process, in addition to the much lower reservoir of vertical kinetic energy we have discussed so far. This could, for example, be caused by internal viscosity provided by magnetic fields, or if oblique shocks take place due to nodal precession associated with black hole spin. As a result, the level of heating would be higher than that predicted from equation (10) and the gas would therefore expand faster from pericenter.

\subsubsection{Self-crossing shock}

Another early dissipation mechanism consists of a self-crossing shock caused by the intersection between the part of the stream that has passed pericenter and the debris still approaching the black hole. The way this collision takes place depends on the following physical effects: (i) relativistic apsidal precession, which modifies the stream trajectory near pericenter to put it on a collision course with the infalling gas, (ii) the expansion resulting from the nozzle shock described in Section 3.1.1, which leads to an increase of the stream width after pericenter passage and (iii) nodal precession induced by the black hole spin, which changes the orbital plane of the gas during pericenter passage. We start by evaluating the influence of these three mechanisms on the self-crossing shock and then describe the gas evolution during the collision for different regimes specified by the relative importance of these effects.

(i) Relativistic apsidal precession: When the stream passes at pericenter, its center of mass precesses by an angle that can be approximated for a nonrotating black hole as (see equation (10.2) of Hobson et al. 2006)

$$
\Delta \phi \approx 3 \pi R_{\mathrm{g}} / R_{\mathrm{p}} \approx 11.5^{\circ} \beta\left(\frac{M_{\mathrm{h}}}{10^{6} \mathrm{M}_{\odot}}\right)^{2 / 3},
$$

where $R_{\mathrm{g}}=G M_{\mathrm{h}} / c^{2}$ is the gravitational radius and the approximation $a_{\min }(1-$ $\left.e_{\min }^{2}\right) \approx 2 R_{\mathrm{p}}$ has been used. If the black hole has a non-zero spin aligned with the angular momentum of the stream, the precession angle is lower than predicted by equation (11). This angle is instead increased in case of antialignment. Apsidal precession by itself can result in a collision between the part 
of the stream moving outward and that still approaching the black hole. ${ }^{8}$ The self-crossing shock taking place when this mechanism dominates is illustrated in the left panel (a) of Fig. 3. Assuming that the two colliding components remain thin with widths $H_{1} \approx H_{2} \ll R_{\text {int }}$, the collision can be considered to happen at a single location. Its characteristics can then be derived analytically by considering two Keplerian ellipses of eccentricity $e_{\min }$ and pericenter $R_{\mathrm{p}}$ rotated according to the above precession angle, as done by several authors (Dai et al. 2015; Jiang et al. 2016b; Bonnerot et al. 2017; Lu and Bonnerot 2019). ${ }^{9}$ The resulting collision occurs at a true anomaly $\theta_{\text {int }}=\pi-\Delta \phi / 2$ that corresponds to an intersection radius of

$$
R_{\mathrm{int}}=\frac{1}{\beta} \frac{R_{\mathrm{t}}\left(1+e_{\mathrm{min}}\right)}{1-e_{\min } \cos (\Delta \phi / 2)},
$$

varying between the apocenter of the most bound debris and its pericenter depending on $\Delta \phi$. The two stream components collide with velocity vectors $\mathbf{v}_{1}^{\text {int }}$ and $\mathbf{v}_{2}^{\text {int }}$ of the same magnitude $v_{\text {int }} \equiv\left|\mathbf{v}_{1}^{\text {int }}\right|=\left|\mathbf{v}_{2}^{\text {int }}\right|$ but inclined by the collision angle $\psi$ given by

$$
\cos \psi=\frac{1-2 e_{\min } \cos (\Delta \phi / 2)+e_{\min }^{2} \cos \Delta \phi}{1-2 e_{\min } \cos (\Delta \phi / 2)+e_{\min }^{2}},
$$

which can be obtained from $\cos \psi=\mathbf{v}_{1}^{\text {int }} \cdot \mathbf{v}_{2}^{\text {int }} / v_{\text {int }}^{2}$ and expressing the two velocities as a function of $R_{\text {int }}$. This angle is small only when apsidal precession is very weak, corresponding to low-mass black holes, i.e. $M_{\mathrm{h}} \lesssim 10^{5} \mathrm{M}_{\odot}$ according to equation (11). Otherwise, the velocity vectors are significantly misaligned, with directions close to being completely opposite with $\psi \approx \pi$. In the typical situation where $M_{\mathrm{h}} \approx 10^{6} \mathrm{M}_{\odot}$ and $\beta \approx 1$, apsidal precession is relatively weak such that the collision happens near apocenter with $R_{\text {int }} \approx 2 a_{\text {min }}$ according to equation (12). For the two stream components to collide instead near pericenter with $R_{\text {int }} \approx R_{\mathrm{p}}$, apsidal precession must be stronger, requiring to increase the black hole mass to $M_{\mathrm{h}} \gtrsim 10^{7} \mathrm{M}_{\odot}$ if $\beta \approx 1$ or the penetration factor to $\beta \gtrsim 5$ if $M_{\mathrm{h}} \approx 10^{6} \mathrm{M}_{\odot}$.

(ii) Expansion from the nozzle shock: As described in Section 3.1.1, dissipation takes place during the strong compression of the stream at pericenter, leading to gas expansion as it then moves away from the black hole. As a result, this part of the stream can be thicker than that still moving inwards, so that the nozzle shock induces an asymmetry between these two components. Due to our limited understanding of the nozzle shock, its impact on the stream width

\footnotetext{
8 Another source of interactions for the stream involves the sequential tidal disruption of the two components of a binary star by a black hole (Bonnerot and Rossi 2019), during which the two debris streams can collide with each other due to the difference in their trajectory induced by the previous binary separation.

9 Note that this treatment is approximate due to the difference in orbital energy between the two gas components involved in the collision. In reality, the part of the stream moving away from the black hole is more bound than the approaching one with a lower apocenter distance.
} 
(a)

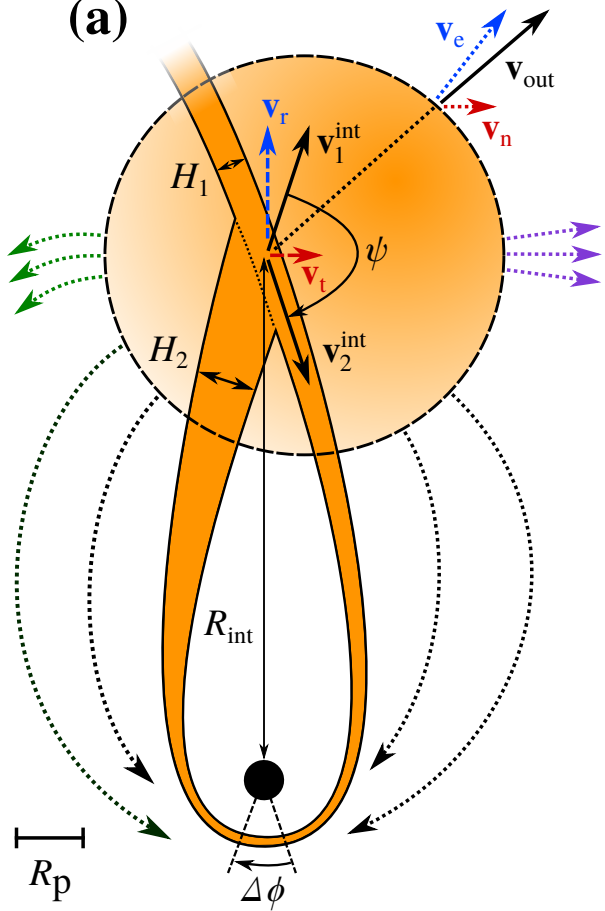

(b)

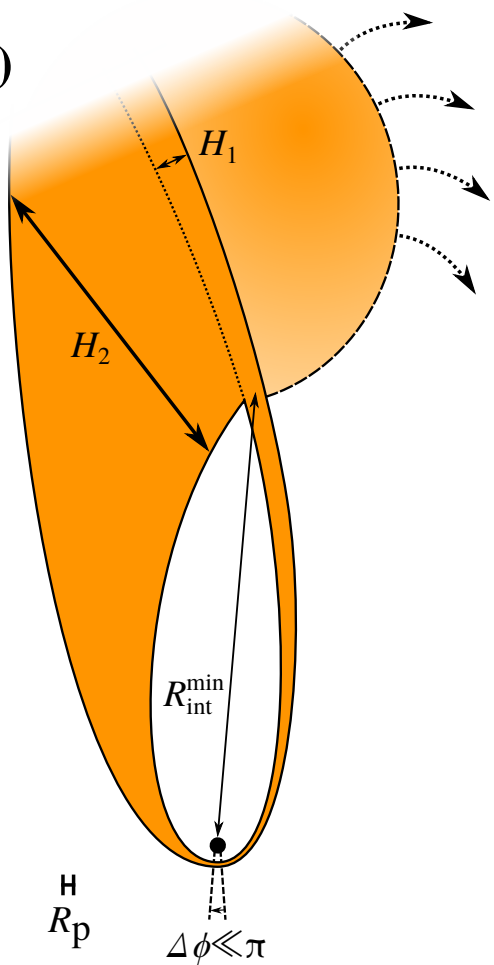

Fig. 3 Sketches representing the self-crossing shock caused by an intersection of the stream with itself, and the subsequent gas evolution in two different regimes. In the left panel (a), the collision is dominated by relativistic apsidal precession, which makes the tip of the stream precess by an angle $\Delta \phi$ during pericenter passage. The resulting intersection takes place at a distance $R_{\text {int }}$ from the black hole, between two stream components assumed to have retained similar widths $H_{1} \approx H_{2} \ll R_{\text {int }}$. Their velocities $\mathbf{v}_{1}^{\text {int }}$ and $\mathbf{v}_{2}^{\text {int }}$ are of the same magnitude but are misaligned by the collision angle $\psi$, such that they have identical tangential components $\mathbf{v}_{\mathbf{t}}$ but opposite radial ones $\pm \mathbf{v}_{\mathrm{r}}$. If the widths of the colliding gas are identical with $H_{1}=H_{2}$ with no vertical offset $\Delta z=0$ between them, all their mass passes through the self-crossing shock, resulting in the dissipation of the kinetic energy associated with the gas radial motion. This causes the formation of an outflow (orange sphere delimited by black dashes) driven by radiation pressure, whose velocity can be decomposed into a net and expansion component as $\mathbf{v}_{\text {out }}=\mathbf{v}_{\mathrm{e}}+\mathbf{v}_{\mathrm{n}}$ where $\left|\mathbf{v}_{\mathrm{e}}\right| \approx\left|\mathbf{v}_{\mathrm{r}}\right|$ and $\mathbf{v}_{\mathrm{n}} \approx \mathbf{v}_{\mathrm{t}}$. Gas expanding in the direction of its net motion (dotted purple arrows) can get unbound from the black hole while that moving in the opposite way (green dotted arrows) can acquire a direction of motion opposite to that of the original star. If the stream components have a slight width difference with $H_{1} \lesssim H_{2}$ or are weakly misaligned with $\Delta z \ll H_{1}+H_{2}$, a small fraction of the gas can avoid the intersection while the rest experiences a collision qualitatively similar to that described above. In the right panel (b), we consider a different limit, wherein the expansion following the nozzle shock produces very different widths for the two stream components, namely $H_{1} \ll H_{2}$. As a result, intersections occur across a broad range of radii even if relativistic precession is negligible $(\Delta \phi \ll \pi)$. Due to the large density difference between the two colliding components, the infalling gas can pass through the outgoing debris with a largely unaffected trajectory. It is possible that a bow shock forms that heats the outward-moving gas causing its mild expansion (orange half-sphere delimited by black dashes) following the intersection. In both regimes, the gas that remains bound after the self-crossing shock eventually returns near the black hole (black dotted arrows) where it can experience additional interactions. 

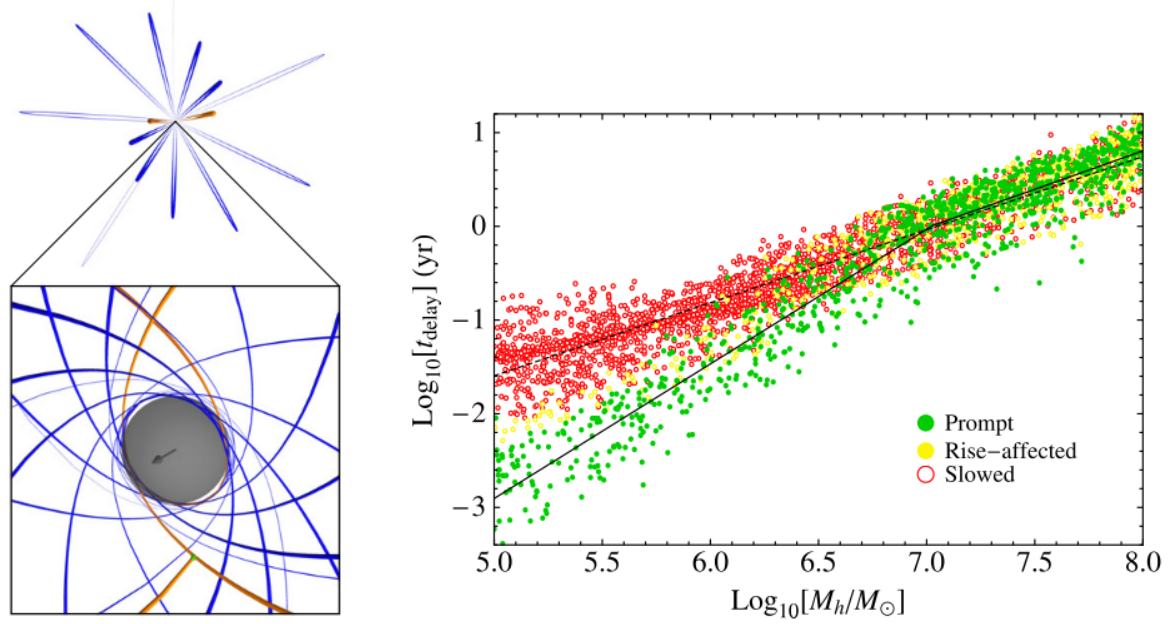

Fig. 4 Left panel: Trajectory of the stream of debris around a spinning black hole (grey sphere) obtained from the semi-analytical model of Guillochon and Ramirez-Ruiz (2015). Because of Lense-Thirring precession, the stream experiences several windings (blue curves) before eventually colliding, as shown with the orange curves that intersect at the green point. Right panel: Time $t_{\text {delay }}$ elapsed between the passage of the star at pericenter and the intersection of the debris stream as a function of black hole mass. Each point corresponds to a different set of parameters assumed for the above process with the colours related to how fast the resulting disc viscously accretes with respect to the time of peak fallback rate.

past pericenter has so far not been precisely quantified. As we explained, the amount of dissipation during this interaction is uncertain, being potentially much larger than current analytical estimates. Additionally, the direction along which the gas preferentially bounces following the compression is largely unknown. It is therefore unclear whether the shocked part of the stream mainly expands within the orbital plane or perpendicular to it. This effect is particularly important since it determines the geometry of the outgoing stream component that may be involved in the self-crossing shock. To take this ambiguity into account, we allow this part of the stream to become thicker than the infalling matter, with $H_{2} \geq H_{1}$ as a result of the nozzle shock. The width ${ }^{10}$ $H_{2}$ can in principle take a wide range of possible values, from $H_{2}=H_{1}$ up to a size comparable to the intersection radius, i.e. $H_{2} \approx R_{\text {int }}$. The left panel (a) of Fig. 3 illustrates the regime where the self-crossing shock is still mainly determined by relativistic apsidal precession, but involves stream components of slightly different widths with $H_{2} \gtrsim H_{1}$. In the right panel (b) of Fig. 3, relativistic precession is negligible and the streams have very different widths, with $H_{1} \ll H_{2} \approx R_{\text {int }}=2 a_{\text {min }}$. The large thickness of the outgoing gas implies that the intersection takes place at a wide range of distances from the black

\footnotetext{
10 Although for simplicity this description involves a single width $H_{2}$ for the outgoing stream component, it is not guaranteed that the gas distribution remains cylindrical following the expansion.
} 
hole. These interactions are indirectly caused by the nozzle shock that deflects a fraction of the gas in the direction of the infalling debris, even in the absence of relativistic precession. A self-crossing shock induced by this effective precession may represent an important dissipation mechanism when relativistic precession is very weak. This scenario seems particularly applicable to black holes with masses $M_{\mathrm{h}} \lesssim 10^{5} M_{\mathrm{h}}$, for which the dynamical impact of the nozzle shock is additionally enhanced, as predicted by the estimates of Section 3.1.1. A few simulations considering intermediate-mass black holes (Ramirez-Ruiz and Rosswog 2009; Guillochon et al. 2014; Shiokawa et al. 2015) have captured this effect but its role in the disc formation process has not been further investigated in a systematic way.

(iii) Spin-induced nodal precession: If the black hole has a non-zero spin, the stream experiences nodal precession (dominated by the Lense-Thirring effect) near pericenter that modifies its orbital plane. As first pointed out by Cannizzo et al. (1990), this effect produces a vertical offset $\Delta z$ between the infalling and outgoing debris at the intersection radius where they would otherwise collide. If $\Delta z<H_{1}+H_{2}$, the self-crossing shock still occurs, but the two stream components are misaligned. The interaction is entirely avoided if $\Delta z>H_{1}+H_{2}$ : in this case, the streams are unable to impact one another, and keep following their trajectories unimpeded. Jiang et al. (2016b) carried out the following analytical estimate to determine whether this initial self-crossing shock is avoided. Nodal precession causes the gas angular momentum vector to precess around the black hole spin direction by an angle $\Delta \Omega \approx 4 \pi 2^{-3 / 2} a \sin i\left(R_{\mathrm{g}} / R_{\mathrm{p}}\right)^{3 / 2} \approx 1^{\circ} a \sin i \beta^{3 / 2}\left(M_{\mathrm{h}} / 10^{6} \mathrm{M}_{\odot}\right)$ (Merritt 2013), where $a$ denotes the dimensionless spin parameter and $i$ represents the angle between the black hole spin and the stellar angular momentum that is generally non-zero. ${ }^{11}$ The resulting shift in orbital plane causes the centers of mass of the two gas components to be separated by a distance of $\Delta z \approx R_{\text {int }} \Delta \Omega$ at the intersection radius given by equation (12). Additionally, the sum of the stream widths at this position is approximated by a single value $H=H_{1}+H_{2} \approx R_{\star} R_{\text {int }} / R_{\mathrm{p}}$ that expands homologously (see Section 2) from pericenter where it is assumed to be of a stellar radius. The ratio of vertical offset to stream width is then given by

$$
\frac{\Delta z}{H} \approx a \sin i \beta^{1 / 2}\left(\frac{M_{\mathrm{h}}}{10^{6} \mathrm{M}_{\odot}}\right)^{4 / 3} .
$$

A prompt collision between misaligned stream components can take place if $\Delta z<H$, that is satisfied for low-mass and slowly-spinning black holes with $M_{\mathrm{h}} \lesssim 10^{6} \mathrm{M}_{\odot}$ and $a \lesssim 1$ if $\beta \approx 1$ and $i \approx 90^{\circ}$ according to this estimate. The intersection is instead completely avoided with $\Delta z>H$ if the black hole has

\footnotetext{
11 As explained in the Rates Chapter, victim stars sourced by two-body relaxation approach the black hole with a quasi-isotropic distribution of orientations, although alternative scenarios (Wernke and Madigan 2019) are possible. As a result, the stellar angular momentum is in general not aligned with the black hole spin: $i \neq 0$.
} 
a larger mass $M_{\mathrm{h}} \gtrsim 10^{7} \mathrm{M}_{\odot}$ and is maximally-spinning with $a \approx 1$, keeping the other parameters fixed. If this early self-crossing shock does not occur, the stream needs to complete additional revolutions around the black hole before eventually colliding with itself. Dai et al. (2013) studied this effect first by integrating the Kerr geodesics followed by the stream. However, the impact of nodal precession was likely overestimated by assuming that the gas is still confined by self-gravity, which is unlikely to be valid near the black hole. A more extensive investigation was done by Guillochon and Ramirez-Ruiz (2015) using a more complex, semi-analytical geodesic model that assumes that the stream width evolves homologously $(H \propto R)$, and also includes the additional expansion induced by the nozzle shock at each pericenter passage. In the regime where prompt self-intersection fails, streams orbiting the black hole will therefore become progressively thicker until two different windings eventually intersect with $\Delta z<H_{1}+H_{2}$, leading to a self-crossing shock. An example of stream evolution obtained from this work is shown in the left panel of Fig. 4 , while the right panel displays the time $t_{\text {delay }}$ between the stellar disruption and the first successful collision as a function of black hole mass. This time delay is typically several orbital periods and increases with black hole mass to reach of order a year for $M_{\mathrm{h}}=10^{7} \mathrm{M}_{\odot}$. A delayed intersection is also more likely to happen between two successive windings, according to this study. In this case, the trajectories of the colliding debris may be close to that of the initial self-crossing shock shown in the left panel (a) of Fig. 3. However, the stream components may have a residual misalignment and widths larger that of the initially returning debris due to the cumulative effect of successive nozzle shocks. Additionally, the cold streams present around the black hole due to previous missed collisions may affect the gas evolution at later times.

Depending on the importance of the effects described above, the subsequent gas evolution can differ significantly. To determine the impact of relativistic precession alone, we start by making the idealized assumption that both the nozzle shock and nodal precession have a negligible impact on the resulting self-crossing shock. The stream components can therefore be approximated as having the same width $H_{1}=H_{2}$ and being perfectly aligned with $\Delta z=0$. In this situation, the collision takes place in a confined region and involves all the mass of the two streams that intersect with opposite radial velocities $\pm \mathbf{v}_{\mathrm{r}}$ as indicated in the left panel (a) of Fig. 3. Because the inflow rate through the collision point is similar ${ }^{12}$ for the two components, the specific energy dissipated by the interaction reaches its maximal value

$$
\Delta \varepsilon_{\mathrm{sc}}^{\max }=\frac{v_{\mathrm{r}}^{2}}{2} \approx \frac{G M_{\mathrm{h}}}{R_{\mathrm{int}}} \sin ^{2}(\psi / 2),
$$

since all the kinetic energy associated with radial motion gets converted into heat. In the second step, the radial velocity is expressed as $v_{\mathrm{r}}=\left|\mathbf{v}_{\mathrm{r}}\right|=$

\footnotetext{
12 Due to the time-dependence of the fallback rate, the inflow rate through the collision point may differ for the two stream components, especially if the intersection happens at large distances with $R_{\text {int }} \gtrsim a_{\text {min }}$.
} 
$v_{\text {int }} \sin (\psi / 2)$ and the gas velocity at the self-crossing shock is approximated by the local escape speed with $v_{\text {int }} \approx\left(2 G M_{\mathrm{h}} / R_{\text {int }}\right)^{1 / 2}$. Except for very low black hole masses, $\psi \approx \pi$, so that a significant fraction of the binding energy at the intersection radius gets dissipated. A dissipated energy $\Delta \varepsilon_{\mathrm{sc}} \approx \Delta \varepsilon_{\mathrm{circ}}$ requires $R_{\text {int }} \approx R_{\mathrm{p}}$, which is only attained for $M_{\mathrm{h}} \gtrsim 10^{7} \mathrm{M}_{\odot}$ or $\beta \gtrsim 5$, as explained above. Only in this situation can we expect a fraction of the gas to reach a significant level of circularization as a result of the first collision, although even in this case, a large fraction of the gas may be unbound from the black hole. Otherwise, relativistic precession is weaker, with $\Delta \phi \ll \pi$, often implying $R_{\mathrm{int}} \approx a_{\min }$ and $\Delta \varepsilon_{\mathrm{sc}} \approx 10^{-2} \Delta \varepsilon_{\mathrm{circ}}$. Following this interaction away from pericenter, most of the gas remains therefore significantly eccentric.

The hydrodynamics of the self-crossing shock has been investigated through local simulations initialized with two identical thin stream components whose properties are obtained analytically (Kim et al. 1999; Jiang et al. 2016b; Lu and Bonnerot 2019). As we explain in Section 3.4, the collision is found to be radiatively inefficient in this limit due to the large optical depths of the confined gas such that the evolution can be accurately approximated as adiabatic. These numerical works demonstrate that the shocked gas gets launched into an outflow driven by radiation pressure. ${ }^{13}$ It can be seen from Fig. 5 that corresponds to the local radiation-hydrodynamics simulation of a strong intersection by Jiang et al. (2016b) where the two thin, intersecting stream components are displayed as orange tubes while the outflow is represented with the blue to yellow surface. An idealized version of this outflowing gas is also illustrated by the orange sphere delimited by black dashes in the left panel (a) of Fig. 3. In addition to this quasi-spherical expansion due the excess internal energy given by equation (15), the gas also retains a net velocity with respect to the black hole inherited from the tangential motion of the colliding streams. The velocity vector of this outflowing matter can therefore be approximately decomposed as $\mathbf{v}_{\text {out }}=\mathbf{v}_{\mathrm{e}}+\mathbf{v}_{\mathrm{n}}$ where $\left|\mathbf{v}_{\mathrm{e}}\right| \approx v_{\mathrm{r}}$ and $\mathbf{v}_{\mathrm{n}} \approx \mathbf{v}_{\mathrm{t}}$, as illustrated in the left panel (a) of Fig. 3. This implies that the gas expanding near the direction of its net motion has a velocity $\left|\mathbf{v}_{\mathrm{n}}\right|+\left|\mathbf{v}_{\mathrm{e}}\right|>\left(\mathbf{v}_{\mathrm{r}}^{2}+\mathbf{v}_{\mathrm{t}}^{2}\right)^{1 / 2}=v_{\text {int }} \approx\left(2 G M_{\mathrm{h}} / R_{\text {int }}\right)^{1 / 2}$ that is larger than the local escape speed (purple dotted arrows). The fraction of debris unbound by the collision becomes significant if the self-crossing shock is strong. For $\beta=1$, unbinding more than $20 \%$ of the colliding gas requires a black hole mass $M_{\mathrm{h}} \gtrsim 5 \times 10^{6} \mathrm{M}_{\odot}$ according to local simulations (Lu and Bonnerot 2019). Similarly, the gas that gets accelerated in the direction opposite to its net speed (green dotted arrows) experiences a change in sign of its angular momentum if $\left|\mathbf{v}_{\mathrm{n}}\right|<\left|\mathbf{v}_{\mathrm{e}}\right|$, so that it ends up rotating in the direction opposite to that of the original star around the black hole.

\footnotetext{
13 The self-crossing shock leads to an increase of the gas temperature to $T_{\mathrm{g}} \approx m_{\mathrm{p}} v_{\mathrm{r}}^{2} / k_{\mathrm{B}} \approx$ $10^{9} \mathrm{~K}$ using equation $(15)$ and a typical radial velocity $v_{\mathrm{r}} \approx 0.01 \mathrm{c}$ for the colliding gas. This shocked matter rapidly cools by emitting photons that results in an increase of radiation energy while that of the gas diminishes. At the end of this process, most of the energy is in the form of photons with an equilibrium temperature of $T_{\mathrm{eq}} \approx\left(\rho_{\mathrm{s}} v_{\mathrm{r}}^{2} / a\right)^{1 / 4} \approx 10^{6} \mathrm{~K}$, where in the lattermost equality we have adopted a density inside the stream of $\rho_{\mathrm{s}} \approx 10^{-7} \mathrm{~g} \mathrm{~cm}^{-3}$ as derived in Section 2 (see Fig. 1). The ratio of gas to radiation pressure is then $P_{\mathrm{g}} / P_{\mathrm{r}} \approx$ $\left(\rho k_{\mathrm{B}} T_{\mathrm{eq}} / m_{\mathrm{p}}\right) /\left(a T_{\mathrm{eq}}^{4} / 3\right) \approx 10^{-3}$
} 


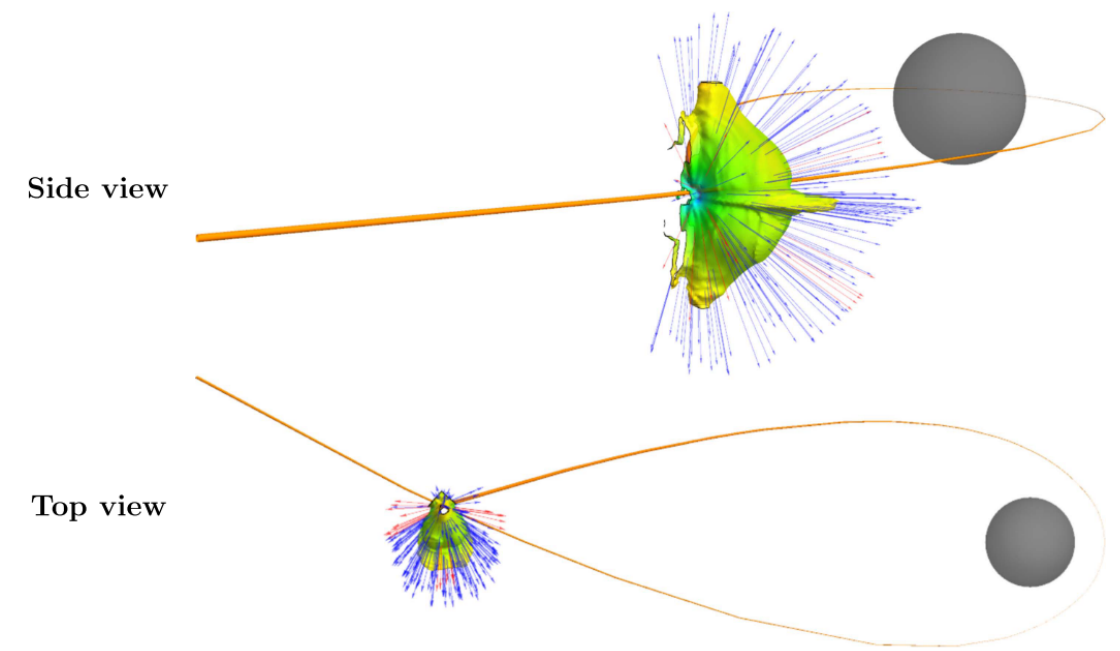

Fig. 5 Gas evolution resulting from the self-crossing shock as obtained from the local radiative transfer simulation of Jiang et al. (2016b) with the two panels adopting different points of view. The stream (orange tube) experiences apsidal precession during its passage close to the black hole (grey sphere) that results in its intersection. Due to the pressure increase, the ensuing self-crossing shock causes the formation of an outflow launched from the intersection point displayed with the blue to yellow surface. The arrows represent the gas velocity for the bound (blue) and unbound (red) matter.

Most of the local studies dedicated to the self-crossing shock have been made in the idealized situation we just discussed. ${ }^{14}$ We present now possible consequences of a more realistic configuration involving two stream components that are misaligned with different widths. For similar widths $H_{1} \lesssim H_{2}$ and a mild vertical offset $\Delta z \ll H_{1}+H_{2}$, only a small fraction of the colliding streams can avoid the interaction. Additionally, the two stream components may reach the intersection point with slightly different inflow rates. These effects tend to reduce the dissipated specific energy below the maximal value of equation (15), i.e. $\Delta \varepsilon_{\mathrm{sc}} \lesssim \Delta \varepsilon_{\mathrm{sc}}^{\max }$. As a result, the shocked gas will expand slower, with less unbound mass, and may evolve into an outflow that deviates from a quasi-spherical wind due to the asymmetry of the collision. Despite these possible differences, the gas evolution is likely to remain qualitatively similar to that predicted above for identical streams. On the other hand, if the widths of the two stream components are very different, with $H_{1} \ll H_{2}$ due to a fast expansion induced by the nozzle shock, most of the outgoing gas can avoid a direct collision. Due to its much larger density, the infalling debris would be largely unaffected by the interaction and act instead as a static obstacle. As a result, a bow shock could form inside the thicker stream com-

\footnotetext{
14 Note that the self-crossing shock found in most global simulations of disc formation presented in Section 3.3 actually differ from the ideal situation of identical stream components, but this difference may, at least partially, originate from the simplified initial conditions used in these studies.
} 
ponent that may cause a slow expansion of this gas when it continues to move outward past the intersection, as is illustrated with the orange half-sphere in panel (b) of Fig. 3. The energy dissipated during this process is likely much lower than that given by equation (15), with $\Delta \varepsilon_{\mathrm{sc}} \ll \Delta \varepsilon_{\mathrm{sc}}^{\max }$, while the radiative efficiency could be significantly higher than mentioned above, as we discuss more in detail in Section 3.4. If the outgoing stream component has a width $H_{2} \approx R_{\text {int }}$ similar to the intersection radius of equation (12), the selfcrossing-shock takes place at a wide range of distances from the black hole due to a deflection of its trajectories induced by the nozzle shock. Despite the overall weak dissipation, a fraction of the debris could collide near pericenter due to a strong effective precession that may result in its circularization. As we mentioned before, this effect could be important for low-mass black holes with $M_{\mathrm{h}} \lesssim 10^{5} \mathrm{M}_{\odot}$. These important differences caused by the increased gas thickness suggest that the hydrodynamics in this regime qualitatively differs from the case of identical streams. However, additional work is needed to better understand the gas evolution during a realistic self-crossing shock.

As we have seen, several possible outcomes are possible for the self-crossing shock depending on how this interaction takes place. It is, however, still largely unclear for which parameters of the problem they are each realised, due to uncertainties in the mechanisms involved, most importantly the expansion at the nozzle shock. Collisions near pericenter are necessary to dissipate a specific energy $\Delta \varepsilon_{\mathrm{sc}} \approx \Delta \epsilon_{\mathrm{circ}}$ but they require either strong relativistic apsidal precession or a large expansion induced by the nozzle shock that only seems realised in specific conditions. In this case, the self-crossing shock itself could result in a significant level of circularization. More generally, interactions happen near the apocenter of the most bound debris with $\Delta \varepsilon_{\text {sc }} \ll \Delta \epsilon_{\text {circ }}$ such that the shocked gas retains large eccentricities. More dissipation would therefore be necessary for the gas trajectories to further circularize. In each regime, the gas remaining bound following the self-crossing shock comes back towards the black hole at later times, as indicated with the dotted arrows in the two panels of Fig. 3. During its subsequent evolution, this matter is likely to experience more interactions, as will be discussed in Section 3.3 based on global simulations.

\subsection{Computational challenges and treatment of general-relativistic effects}

In Section 3.1, we described the early dynamics followed by the debris based on analytical arguments and local numerical studies of the self-crossing shock that apply for the realistic situation of a star disrupted on a parabolic orbit by a supermassive black hole. Studying the more complex evolution of this matter at later times requires to perform a global simulation of the disc formation process. However, this task turns out to be very numerically challenging for the following reasons. The fluid elements inside the stream have semi-major axes larger than that of the most bound debris $a_{\text {min }}$ while the returning matter evolves on scales similar to $R_{\mathrm{p}} \ll a_{\min }$ that are two to three orders of magnitude smaller. The computational cost associated with this large dynamic 
range is exacerbated by the high aspect ratio of the stream due to the transverse confinement by self-gravity that limits its width to $H \approx 10 R_{\star} \ll R_{\mathrm{p}}$ (see Section 2.1). This numerical challenge has long been recognized. The first hydrodynamical simulation of the disc formation process by Ayal et al. (2000) was realised for a realistic setup using a post-Newtonian smoothed-particle hydrodynamics technique. However, it is now clear that the particle resolution used in this work is insufficient to accurately follow the gas evolution near pericenter, leading to important numerical artefacts such as the unbinding of most of the returning stream. Table 1 contains an exhaustive list of more recent numerical studies along with the parameters they use and the method employed to account for general-relativistic effects. In order to have sufficient resolution, most of these works had to adopt simplified initial conditions that are not astrophysically realistic. Two different "compromises" have been used that aim at artificially decreasing the dynamic range of the problem to make it computationally tractable. More recently, Bonnerot and $\mathrm{Lu}$ (2019) used a third strategy that consists in injecting gas into the computational domain to model the outflow resulting from the self-crossing shock. This method allowed them to perform for the first time a simulation of disc formation for realistic parameters of the problem. We start by describing these approaches below along with the astrophysical limitations of each and then focus on the treatment of general relativistic effects. A detailed discussion of different numerical methods can be found in the Simulation Methods Chapter while the Future Modeling Chapter describes innovative techniques that could be used in the future.

The first compromise is to simulate the disruption of a star by an intermediatemass black hole rather than a supermassive one. Since $a_{\min } / R_{\mathrm{t}} \propto M_{\mathrm{h}}^{1 / 3}$ (see equations (1) and (3)), the dynamic range may be reduced by an order of magnitude by considering a black hole mass of $M_{\mathrm{h}}=10^{3} M_{\odot}$ instead of a more realistic choice of $M_{\mathrm{h}}=10^{6} \mathrm{M}_{\odot}$. The first such simulations (Rosswog et al. 2008; Ramirez-Ruiz and Rosswog 2009; Rosswog et al. 2009) were carried out to study tidal disruptions by intermediate-mass black holes for themselves. One important motivation is that white dwarves can only be disrupted outside the event horizon of the black hole for $M_{\mathrm{h}} \lesssim 10^{5} \mathrm{M}_{\odot}$. Later numerical works (Guillochon et al. 2014; Shiokawa et al. 2015) were instead performed with the intention to get insight into the case of a supermassive black hole. However, disruptions by such low-mass black holes differ from the realistic situation in three main ways that must be kept in mind when attempting to extrapolate the results: (i) the dynamical importance of energy dissipation at the nozzle shock is overestimated as shown in Section 3.1.1 (Guillochon et al. 2014), (ii) the pericenter distance is less relativistic since $R_{\mathrm{t}} / R_{\mathrm{g}} \propto M_{\mathrm{h}}^{-2 / 3}$ that can reduce the apsidal precession angle ${ }^{15}$ and strength of the self-crossing shock (see Section 3.1.2) and (iii) the tidal approximation is itself less generally well-

\footnotetext{
15 Relativistic apsidal precession can be important for TDEs involving intermediate-mass black holes if $\beta \gg 1$ but this type of deeply-penetrating encounter is uncommon and poses its own computational challenges related to accurately resolving mid-plane compression in the disrupting star and the returning debris streams.
} 
satisfied as $R_{\mathrm{t}} / R_{\star} \propto M_{\mathrm{h}}^{1 / 3}$. Of these three effects, (i) and (ii) may be quite important for the process of disc formation. It is less likely that (iii) has direct relevance for this phase of evolution, although it may result in a more subtle shift in the energy distribution of the debris (Brassart and Luminet 2008).

The second numerical compromise that has been made is to simulate the disruption by a supermassive black hole of a star on an initially bound rather than parabolic trajectory. The associated decrease in the semi-major axis $a_{\text {min }}$ of the most-bound debris can significantly reduce the numerical cost of the simulation for stellar eccentricities of $e_{\star} \lesssim 0.95$. Although mechanisms exist (e.g. Amaro-Seoane et al. 2012; Stone and Loeb 2012) that can lead to stellar disruptions on bound orbits, the main motivation for carrying out such simulations is to extrapolate the results to the realistic case of an initial parabolic trajectory. This technique was first used by Hayasaki et al. (2013) and later in several other investigations (Bonnerot et al. 2016; Hayasaki et al. 2016; Sadowski et al. 2016). Although this approach does not suffer from the issues listed above for the first method since it uses the correct black hole mass, it has its own disadvantages: (i) the stream produced by the disruption of a bound star is artificially truncated and, if $e_{\star}$ is too small, one winds up in the unrealistic situation where the "head" of the stream must fruitlessly chase its "tail" for several orbits before any major interaction takes place and (ii) the debris stream produced by the disruption process is artificially bound with an apocenter much less than $a_{\text {min }}$, which artificially enhances the strength of the self-crossing shock.

The strategy designed by Bonnerot and Lu (2019) consists in modelling the outflow from the self-crossing shock by an injection of gas into the computational domain. This method is promising since it can be applied for both a parabolic orbit of the star and a supermassive black hole mass. Nevertheless, the idealized treatment of the self-crossing shock implies the following main caveats: (i) the properties of this interaction are fixed, which neglects any feedback resulting from the formation of the disc such as its impact on the stream trajectory near pericenter and (ii) this strategy requires that the self-crossing shock can be treated as a local interaction, which may not be possible if one of the colliding stream components has a width similar to the intersection radius (see right panel of Fig. 3).

General-relativistic effects are essential to properly describe the gas dynamics during disc formation and must therefore be taken into account to simulate this phase of evolution. In particular, this process is thought to be initiated by a self-crossing shock (see Section 3.1.2) that depends on both relativistic apsidal and nodal precession, the latter being induced by the black hole spin. Despite the large computational cost of this approach, some numerical works (e.g. Shiokawa et al. 2015; Sadowski et al. 2016) adopt a fully generalrelativistic treatment. To reduce this numerical expense, other investigations take these effects into account through the use of gravitational potentials that differ from the usual Keplerian one of $\Phi=-G M_{\mathrm{h}} / R$ while still using a Newto- 


\begin{tabular}{|c|c|c|c|c|c|}
\hline Reference & $M_{\mathrm{h}}\left(\mathrm{M}_{\odot}\right)$ & $a$ & $e_{\star}$ & $\beta$ & Method \\
\hline Ramirez-Ruiz et al. $(2009)^{\dagger}$ & $10^{3}$ & 0 & 1 & 3 & $\mathrm{~N}$ \\
\hline Rosswog et al. $(2009)^{\dagger}$ & $100-10^{4}$ & 0 & 1 & $0.9-12$ & PNP \\
\hline Haas et al. $(2012)^{\dagger}$ & $10^{3}$ & $0-0.6$ & 1 & $6-8$ & full GR \\
\hline Hayasaki et al. (2013) & $10^{6}$ & 0 & 0.8 & 5 & PNP \\
\hline Guillochon et al. (2014) & $10^{3}$ & 0 & 1 & 1 & $\mathrm{~N}$ \\
\hline Shiokawa et al. (2015) & 500 & 0 & 1 & 1 & full GR \\
\hline Evans et al. $(2015)^{\dagger}$ & $10^{5}$ & 0 & 1 & $10-15$ & full GR \\
\hline Bonnerot et al. (2016) & $10^{6}$ & 0 & $0.8-0.95$ & $1-5$ & PNP \\
\hline Hayasaki et al. (2016) & $10^{6}$ & $-0.9-0.9$ & 0.8 & $1-5$ & $\mathrm{PNC}$ \\
\hline Sadowski et al. (2016) & $10^{5}$ & 0 & 0.97 & 10 & full GR \\
\hline Bonnerot and Lu (2019) & $2.5 \times 10^{6}$ & 0 & 1 & 1 & PNP \\
\hline Liptai et al. (2019) & $10^{6}$ & $-0.99-0.99$ & 0.95 & $1-5$ & full GR \\
\hline
\end{tabular}

Table 1 List of the numerical works investigating the disc formation process in TDEs. The majority of these simulations make use of adiabatic equations of state. ' $N$ ' stands for Newtonian gravity, 'PNP' for pseudo-Newtonian potential, 'PNC' for post-Newtonian corrections. The references with the superscript $\dagger$ explicitly aim at studying disruptions by intermediate-mass black holes while the others with low black hole masses attempt to extrapolate their result to the case of a supermassive black hole.

nian description of the hydrodynamics. ${ }^{16}$ The first approach adopted by several authors (Rosswog et al. 2009; Hayasaki et al. 2013; Bonnerot et al. 2016) is to use so called pseudo-Newtonian potentials that are designed by hand to reproduce certain features of general-relativistic gravity. Popular choices include the famous Paczynski-Wiita potential (Paczyńsky and Wiita 1980) and those designed by Wegg and Bode (2011) and Tejeda and Rosswog (2013). The latter two are especially relevant since they are tailored to reproduce apsidal precession rates in a Schwarzschild spacetime. A second option is to use post-Newtonian potentials (e.g. Blanchet 2014), which are self-consistently derived from the low-velocity limit of general relativity. This is the approach that was used in the first simulations of mass return in TDEs (Ayal et al. 2000). However, the appeal of self-consistency is often outweighed by the poor convergence of the post-Newtonian approximation as distances approach $R_{\mathrm{g}}$. Test particle dynamics in such potentials are increasingly untrustworthy at $R \lesssim 15 R_{\mathrm{g}}$, where large deviations from general-relativistic geodesics occur, although it is a good approximation at larger scales. The main advantage of this approach is that it offers a well-defined way to incorporate nodal precession due to black hole spin (Faye et al. 2006) that has been exploited by Hayasaki et al. (2016).

\subsection{Global hydrodynamics of accretion flow formation}

As explained in Section 3.2, it is not yet computationally feasible to perform a global simulation of disc formation for realistic initial conditions. The nu-

\footnotetext{
16 Note that several investigations (Guillochon et al. 2014; Bonnerot et al. 2016) nevertheless adopt an entirely Keplerian description of the dynamics as an attempt to pinpoint purely relativistic features.
} 
merical works listed in Table 1 therefore rely on computationally-motivated idealizations to make the problem tractable. In most of them, this is achieved by either considering an intermediate-mass black hole or an initially bound star. More recently, Bonnerot and $\mathrm{Lu}$ (2019) were able to consider realistic astrophysical parameters of the problem using another simplifying strategy: treating the self-crossing shock as an injection of gas into the computational domain. Most of these works adopt an adiabatic equation of state for the gas while a minority of others (e.g. Hayasaki et al. 2013) assumes that the debris evolves instead isothermally. The adiabatic choice is motivated by the radiative inefficiency of the self-crossing shock if it involves thin stream components (see Section 3.1.2) but it is unclear whether this assumption remains acceptable in the later stages of disc formation. As we discuss in Section 3.4, it is possible that the circularizing shocks become more radiatively efficient as the accretion flow starts forming, with the hydrodynamics significantly deviating from complete adiabaticity. In the following, we describe the ways in which the simulated debris evolves towards an accretion flow, and the properties of the newly-formed discs obtained from these simulations. All these works find that a majority of the bound gas has its average eccentricity decreased due to the interactions it experiences, resulting in a more circular gas distribution. We are particularly interested in the "circularization timescale," $t_{\text {circ }}$, required for this process to complete, keeping in mind that the resulting accretion flow is in general not entirely circular with the gas retaining instead significant eccentricities. Because these simulations use idealized initial conditions, it is not a simple task to extrapolate from these numerical results to the astrophysically realistic situation. We attempt to highlight when this seems nevertheless possible and mention possible differences if such a generalization appears unreliable.

The first hydrodynamic simulation following accretion flow formation in its entirety is that by Hayasaki et al. (2013), who consider a bound star with $e_{\star}=0.8$ and $\beta=5$ disrupted by a supermassive black hole of mass $M_{\mathrm{h}}=10^{6} \mathrm{M}_{\odot}$. Due to the low stellar eccentricity, all the debris is bound, unlike the situation in a parabolic encounter. As explained in Section 3.2, this causes the stream to chase its "tail" for a few orbits before it first intersects itself due to strong relativistic apsidal precession. Collisions near pericenter then happen at each revolution, although the first ones only involve the stream's low-density extremities. As a result, an accretion disc forms on a timescale $t_{\text {circ }} \approx 5 P_{\star}$ where $P_{\star}$ denotes the stellar period..$^{17}$ Later simulations (Bonnerot et al. 2016; Hayasaki et al. 2016) explored the impact of the stellar trajectory and gas thermodynamics on this process. They find that an increase in eccentricity to $e_{\star} \approx 0.95$ allows the stream to promptly cross itself for $\beta=5$ without the prior revolutions artificially needed for a more bound star. Due

\footnotetext{
17 Because of the bound stellar trajectory, the difference in period between the different parts of the stream is negligible compared to the period of the star. For this reason, when discussing idealized simulations with significantly sub-parabolic stellar eccentricities, we express the circularization timescale in terms of the stellar period rather than that of the most bound debris.
} 


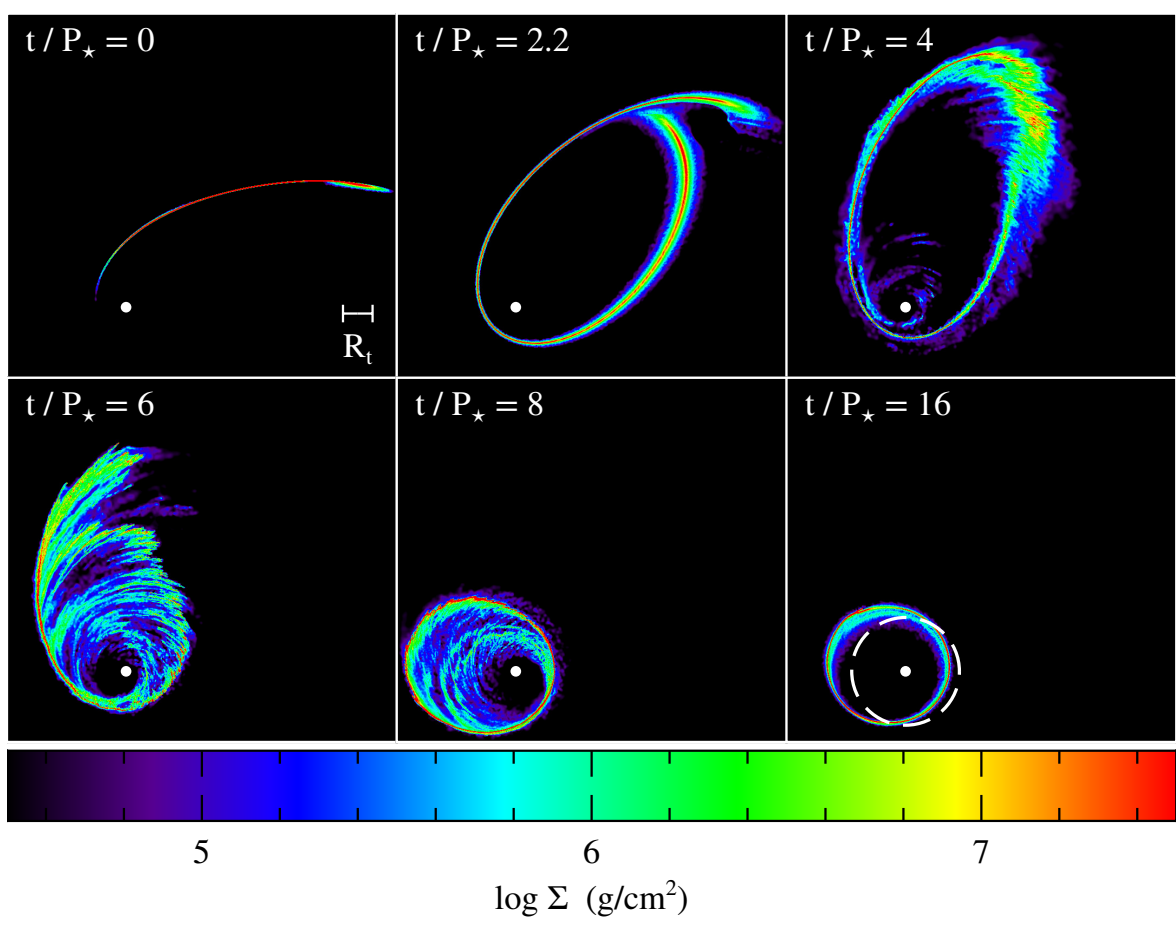

Fig. 6 Snapshots showing the evolution of column density during the disc formation process simulated by Bonnerot et al. (2016), considering the disruption of a solar-type star on a trajectory with eccentricity $e_{\star}=0.8$ and penetration factor $\beta=1$ by a supermassive black hole of mass $M_{\mathrm{h}}=10^{6} \mathrm{M}_{\odot}$. Due to weak relativistic precession, the stream intersects itself near apocenter, and the debris circularizes on a timescale $t_{\mathrm{circ}} \approx 10 P_{\star}$. Importantly, the gas is assumed to evolve isothermally, resulting in the formation of a thin ring located around the circularization radius $R_{\text {circ }} \approx 2 R_{\mathrm{p}}$ (dashed circle in the bottom right snapshot).
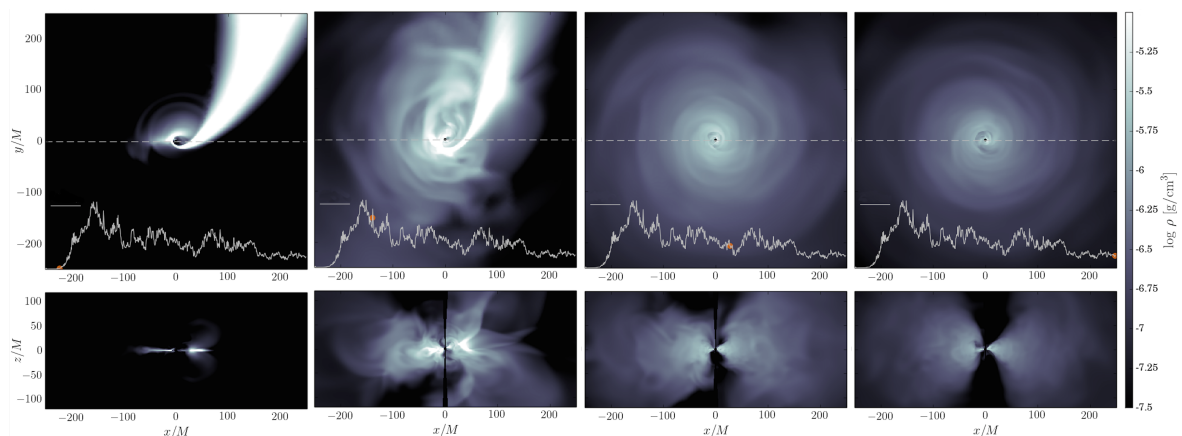

Fig. 7 Snapshots from the simulation by Sadowski et al. (2016), that studied the disc formation process for a star with eccentricity of $e_{\star}=0.97$ and penetration factor $\beta=10$, disrupted by a black hole of mass $M_{\mathrm{h}}=10^{5} \mathrm{M}_{\odot}$. The density distribution is shown in slices parallel (upper panels) and perpendicular (lower panel) to the stellar orbital plane. Due to strong relativistic precession, a self-crossing shock takes place near pericenter, resulting in a fast outflow and the formation of an accretion flow on a timescale $t_{\text {circ }} \approx 5 P_{\star} \ll t_{\text {min }}$. Because the gas is assumed to evolve adiabatically, the resulting disc is extended and thick 
to the large precession angle, the ensuing self-crossing shock is strong, causing rapid formation of an accretion disc on a reduced timescale of $t_{\text {circ }} \approx P_{\star}$. However, decreasing the penetration factor to a more common value of $\beta=1$ results in collisions near the apocenter of the stream due to the decreased relativistic precession angle, as described in Section 3.1.2. As can be seen from Fig. 6 , these weak interactions result in a slower disc formation with $t_{\text {circ }} \approx 10 P_{\star}$. As in the earlier work by Hayasaki et al. (2013), this evolution is somewhat delayed by the bound stellar trajectory considered. The gas also does not significantly expand during the circularizing shocks, causing the formation of a thin circular ring at $R_{\text {circ }} \approx 2 R_{\mathrm{p}}$. Importantly, this outcome directly results from the assumed isothermal equation of state, that allows the gas to effectively lose energy at shocks. As emphasized above, this assumption is unlikely to be valid in most cases due to the large optical thickness of the colliding streams. By modifying the equation of state to the more physical adiabatic limit, Hayasaki et al. (2016) and Bonnerot et al. (2016) find that the stream expands as a result of collisions. However, this additional expansion does not unbind any gas from the black hole, which is likely due to the artificially bound stellar orbit considered. In this case, the resulting accretion disc is thick and occupies a wide range of radii centred around the stellar semi-major axis.

Sadowski et al. (2016) also studied disc formation assuming adiabaticity for an eccentric stellar trajectory, with $e_{\star}=0.97$ and a large penetration factor of $\beta=10$. As can be seen from the snapshots of Fig. 7, strong relativistic precession then results in a self-crossing shock near pericenter. This collision involves stream components of different widths with $H_{2} \gtrsim H_{1}$ (see Fig. 3) due to a fast expansion caused by the nozzle shock during pericenter passage, as discussed in Section 3.1.1. Despite this asymmetry, the collision is powerful enough to cause the shocked gas to expand into an outflow whose unbound component represents about $15 \%$ of the stellar matter. While this unbinding of matter is consistent with the expectation of Section 3.1.2, the gas retains its original direction of rotation around the black hole, in contrast to the change of angular momentum sign predicted by local studies for a strong self-crossing shock. The bound part of this post-shock gas then returns near the black hole with a significant fraction being ballistically accreted along the funnels of the forming disc. After experiencing numerous interactions, the debris settles on a timescale $t_{\text {circ }} \approx 5 P_{\star}$ into an extended and thick accretion flow. As in the prior works that assume adiabaticity, this disc extends roughly to the stellar semi-major axis, with significant pressure support against gravity in its outer region. Despite its overall axisymmetric structure, the gas retains significant eccentricities with $e \approx 0.2$ on average. This work was also the first to include gas magnetic fields, but these were found not to modify the hydrodynamics, as we discuss more in detail in Section 3.5.3. In this study as well as the above simulations with $e \gtrsim 0.95$ and $\beta \gtrsim 5$ (e.g. Bonnerot et al. 2016) that are able to capture a prompt self-crossing shock, the gas evolution may be comparable to that of a deep parabolic encounter, although the precise properties of the initial collision could significantly differ (see Section 3.1.2). Because the reduced period of the stream is washed out by the large redistribution of or- 

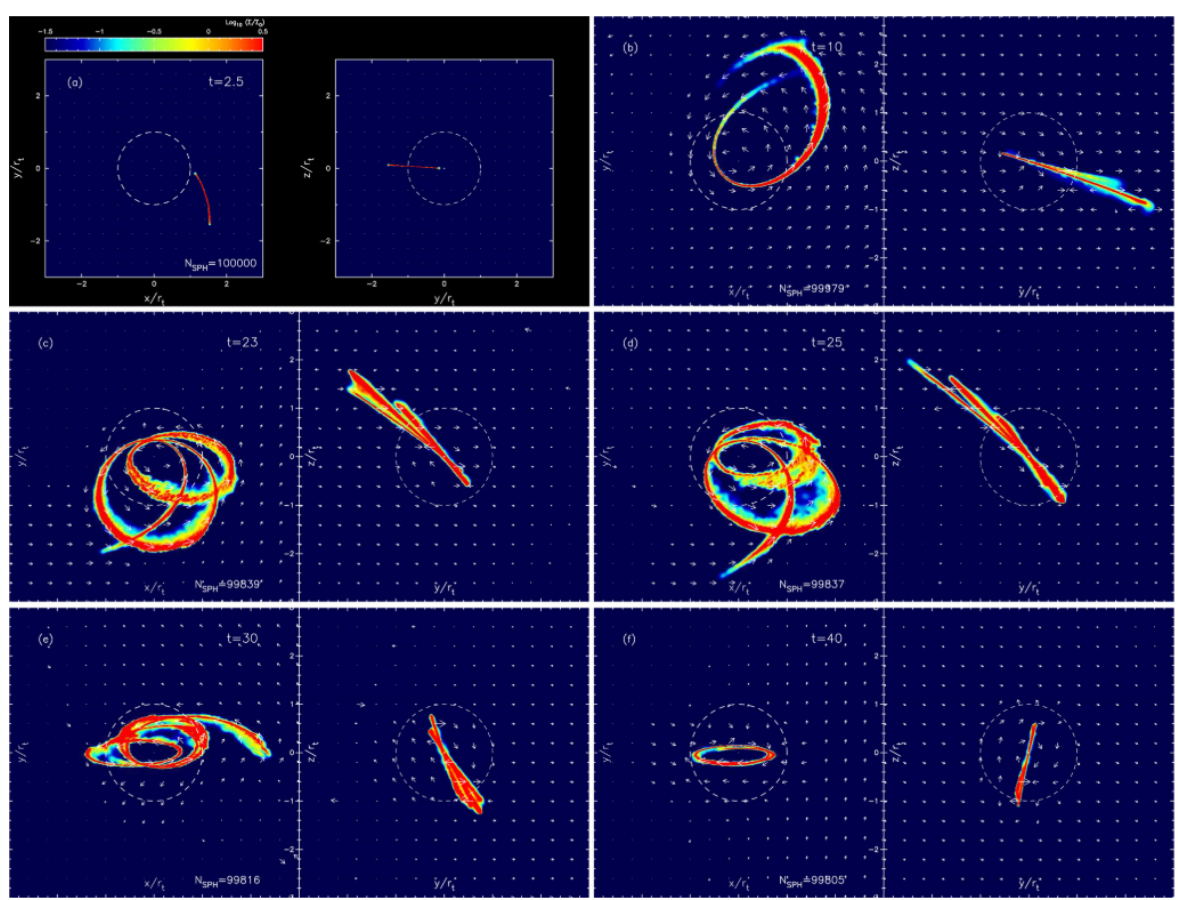

Fig. 8 Snapshots from the simulation by Hayasaki et al. (2016) who study the disc formation process for a star with initial eccentricity of $e_{\star}=0.7$ disrupted by a black hole with spin $a=0.9$ inclined with respect to the stellar orbital plane by an angle $i=90^{\circ}$. Because the gas is assumed to evolve isothermally, it does not expand significantly during pericenter passage such that nodal precession prevents a successful self-crossing shock for several orbits. Interactions eventually take place that cause the delayed formation of a narrow accretion disc on a timescale $t_{\text {circ }} \gtrsim 10 P_{\star}$.

bital elements induced by multiple collisions, it appears legitimate to compare the circularization timescale of a few $P_{\star}$ found in this regime to the period $t_{\min }$ of the most bound debris assuming a parabolic stellar trajectory. This comparison yields $t_{\text {circ }} \lesssim t_{\text {min }}$, which suggests that disc formation happens rapidly for deep encounters where a strong self-crossing shock takes place. ${ }^{18}$ For $\beta \approx 1$, extrapolating eccentric disruption results to the parabolic case is more uncertain due to the reduced size of an entirely bound stream, which can have a larger influence on the gas evolution.

\footnotetext{
18 A more extreme situation is that of an ultra-deep encounter where the stellar pericenter is similar to the gravitational radius of the black hole. In this case, the disruption of the star is accompanied by a stretching of the debris into a elongated structure. Due to the very large values of the apsidal precession angle with $\Delta \phi \gtrsim \pi$, it is possible that this gas collides with itself during the first passage of the star near pericenter. Numerical investigations of this process (Haas et al. 2012; Evans et al. 2015; Darbha et al. 2019) find that this early selfcrossing shock results in the fast formation of an accretion flow around the black hole. We note however that such relativistic pericenters are rare for black holes substantially smaller than the Hills mass.
} 


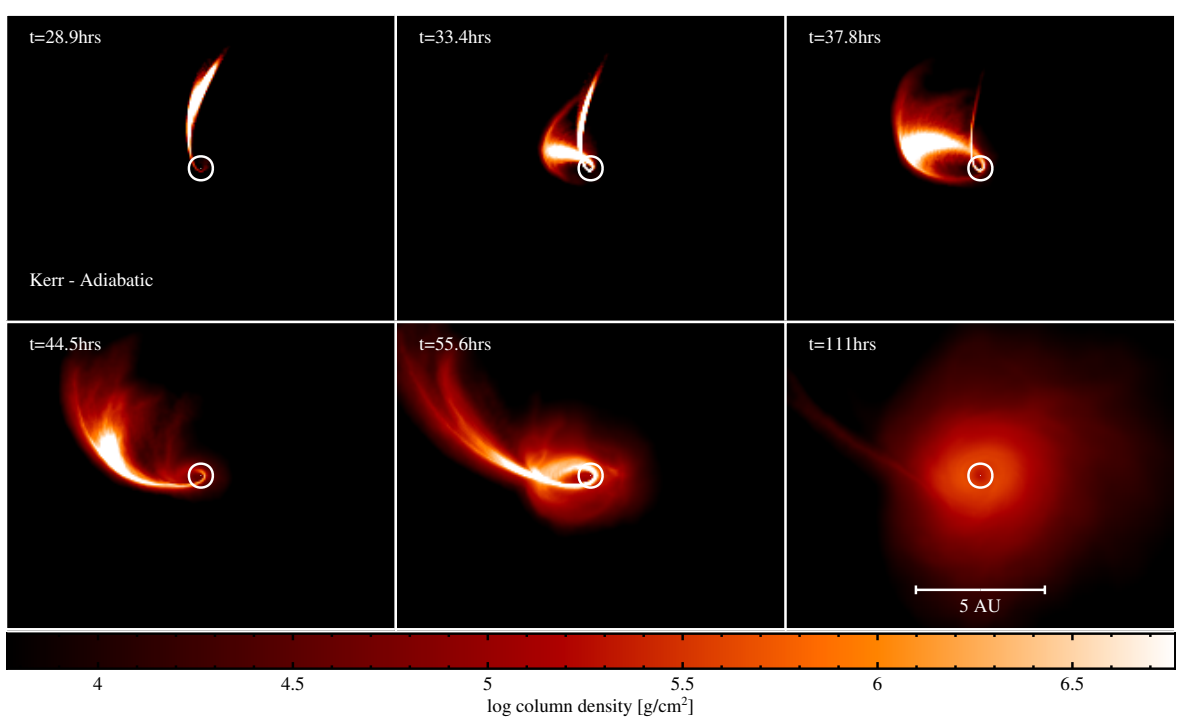

Fig. 9 Snapshots from the simulation of Liptai et al. (2019), that studies the disc formation process for a star with eccentricity $e_{\star}=0.95$ and penetration factor $\beta=5$ disrupted by a black hole with spin $a=0.99$ inclined with respect to the stellar orbital plane by an angle $i=60^{\circ}$. Nodal precession produces a vertical offset that prevents the stream from colliding with itself promptly after its first pericenter passage. However, due to the adiabatic equation of state assumed for the gas, the nozzle shock results in a significant expansion that causes a successful self-crossing shock during the next orbit. This interaction results in the formation of a thick and extended accretion disc that is only mildly delayed, with a circularization timescale $t_{\text {circ }} \lesssim 5 P_{\star}$

The influence of black hole spin has also been studied in simulations considering initially bound stars and different equations of state (Hayasaki et al. 2016; Liptai et al. 2019). When the gas is assumed to evolve isothermally, the energy dissipated by the nozzle shock is removed, so that there is no significant expansion of the stream during pericenter passage. In this case, nodal precession causes a vertical offset $\Delta z>H_{1}+H_{2}$ (see Section 3.1.2) that prevents the stream from colliding with itself even after multiple orbits. This effect is illustrated in Fig. 8 that shows the gas evolution obtained by Hayasaki et al. (2016) for a star with $e_{\star} \approx 0.7$ and $\beta=2$, disrupted by a black hole with spin $a=0.9$, and inclined by an angle $i=90^{\circ}$ with respect to the orbital plane. A "wicker basket" configuration is created as the stream wraps around the black hole until a self-crossing shock eventually takes place causing disc formation to complete after tens of dynamical times with $t_{\text {circ }} \gtrsim 10 P_{\star}$. These works also find that the nozzle shock makes the stream expand faster when adiabaticity is assumed, which is likely a more physical choice given the large optical depth of the confined gas. In this limit, the delay of the first collision is reduced due to the increase in stream width that can rapidly overcome the vertical offset induced by nodal precession, i.e. $\Delta z<H_{1}+H_{2}$. As a result, no delay to the self-crossing shock is observed in the simulations by Hayasaki et al. (2016) for 
the parameters they consider. The more recent investigation by Liptai et al. (2019) finds a similar gas evolution, shown in Fig. 9 for a stellar trajectory with $e_{\star}=0.95$ and $\beta=5$, a black hole spin $a=0.99$, and an inclination angle $i=60^{\circ}$. It can be seen that nodal precession prevents the stream from colliding with itself shortly after its first pericenter passage. However, the fast gas expansion caused by the nozzle shock results in a self-crossing shock during the second orbit. Later on, this interaction induces the formation of a thick accretion disc, that is only mildly delayed with $t_{\text {circ }} \lesssim 5 P_{\star}$. This conclusion appears qualitatively different from that obtained by Guillochon and RamirezRuiz (2015) in the semi-analytical work discussed in Section 3.1.2, which found that the first collision usually takes place after a large delay of multiple orbital timescales, even when stream expansion near pericenter is taken into account. The discrepancy could be due to an artificially small amount of stream expansion in the analytic model for the nozzle shock compared to that found in simulations ${ }^{19}$ or alternatively to the unrealistically low eccentricities of the debris resulting from the bound stellar trajectory.

The recent simulation by Bonnerot and Lu (2019) uses the outflow launched from the self-crossing shock as an initial condition by artificially injecting gas inside the computational domain from the intersection point. Using this numerical strategy, this work is able to consider realistic parameters of the problem: a parabolic stellar trajectory and a supermassive black hole of mass $M_{\mathrm{h}}=2.5 \times 10^{6} \mathrm{M}_{\odot}$. The properties of the injected matter are obtained from a local numerical study of the collision ( $\mathrm{Lu}$ and Bonnerot 2019) assuming that the two stream components have identical and thin widths with $H_{1}=H_{2} \ll R_{\text {int }}$ (see left panel (a) of Fig. 3). For their choice of parameters, the stream crosses itself relatively close to pericenter with an intersection radius of $R_{\text {int }} \approx 25 R_{\mathrm{p}}$. The ensuing self-crossing shock is strong, leading to the unbinding of about $33 \%$ of the initially bound gas via the mechanism described in Section 3.1.2, while most of the remaining bound matter has a sign of angular momentum opposite to that of the original star. This gas quickly expands into an envelope that completely engulfs the black hole, as can be seen from the snapshots of Fig. 10 obtained from this simulation. As its trajectories intersect, multiple secondary shocks take place that cause the rapid formation of an accretion disc on a timescale of $t_{\text {circ }} \approx 0.3 t_{\text {min }}$. The resulting accretion disc is thick, with significant pressure support due to the adiabatic equation of state used, and retains an average eccentricity of $e \approx 0.2$. The disc is located within a radius $R_{\mathrm{d}} \approx 15 R_{\mathrm{p}}$ that contains only a small fraction of the injected gas. Due to the angular momentum sign of the injected bound debris, the accretion flow produced from this matter also rotates in the retrograde direction compared to the star. Additionally, it features two spiral shocks that drive a slow inflow along the disc mid-plane, although most of the accretion onto the black hole is due to gas falling along the funnels of the disc as it assembles. Despite the realistic astrophysical parameters it considers,

\footnotetext{
19 This difference could originate from the non-zero black hole spin, that may significantly increase the energy dissipated during the nozzle shock if nodal precession causes the formation of oblique collisions, as proposed in Section 3.1.1.
} 


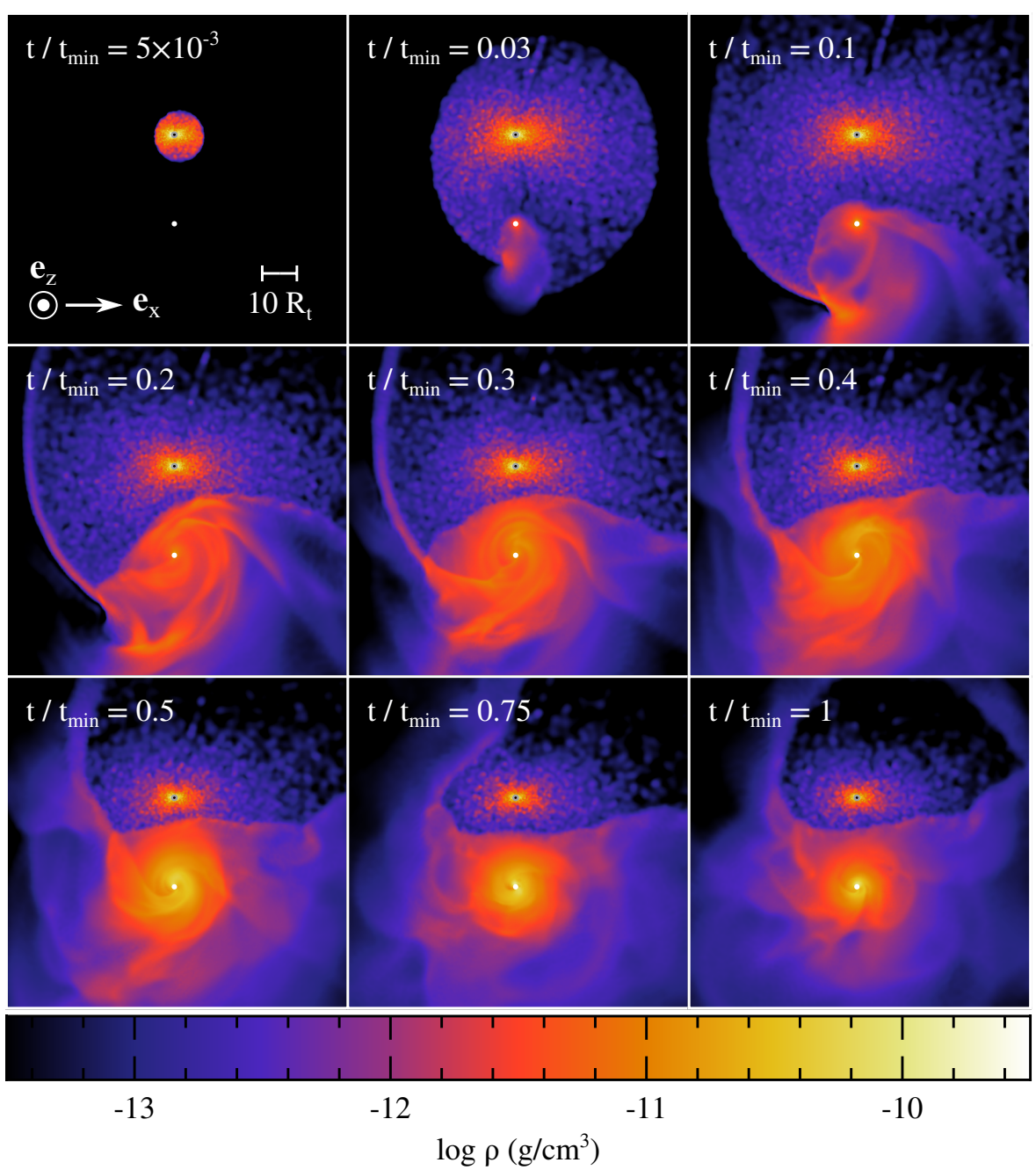

Fig. 10 Snapshots showing the gas evolution in a slice parallel to the orbital plane of the star during the disc formation process simulated by Bonnerot and Lu (2019) for a parabolic stellar trajectory and a supermassive black hole of mass $M_{\mathrm{h}}=2.5 \times 10^{6} \mathrm{M}_{\odot}$. This numerical work uses as initial condition the outflow launched from a strong self-crossing shock whose properties are obtained from a local study of the collision ( $\mathrm{Lu}$ and Bonnerot 2019). Gas is injected inside the computational domain from the intersection point (grey circle) located at $R_{\mathrm{int}} \approx 25 R_{\mathrm{t}}$. This outflowing matter rapidly engulfs the black hole and experiences multiple interactions that result in the formation of a thick and extended accretion disc on a timescale $t_{\text {circ }} \approx 0.3 t_{\text {min }}$ 

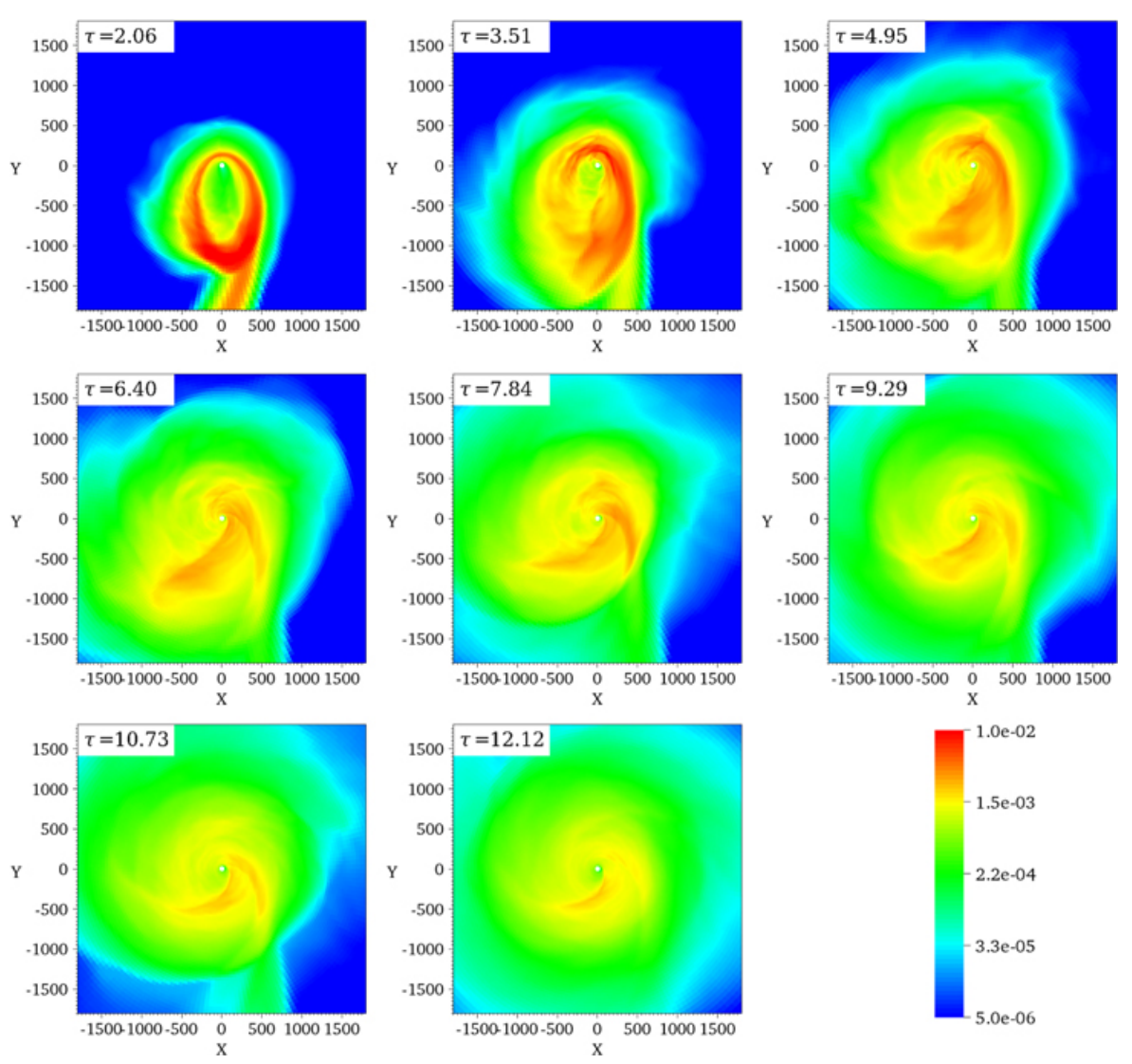

Fig. 11 Snapshots showing the column density evolution during the disc formation process in the simulation carried out by Shiokawa et al. (2015) that consider the disruption of a white dwarf on a parabolic orbit by an intermediate-mass black hole of mass $M_{\mathrm{h}}=500 \mathrm{M}_{\odot}$. In the first snapshot, the tip of the stream has already passed pericenter and intersects with the matter still moving inward that results in a self-crossing shock near apocenter. This weak collision initiates the formation of an accretion flow that remains globally eccentric for several orbital periods before eventually settling on a timescale of $t_{\mathrm{circ}} \approx 10 t_{\min }$ into a more axisymmetric, thick and extended structure.

this simulation remains simplified due to its idealized treatment of the selfcrossing shock by an injection of gas, as explained in Section 3.2. In particular, it is unclear whether this collision actually results in the large outflow used as initial conditions, which closely relates to uncertainties mentioned in Section 3.1.2 regarding this early source of dissipation. Nevertheless, the similarity of this work with those presented above that self-consistently simulate a strong self-crossing (e.g. Sadowski et al. 2016) seems to suggest that the gas evolution is qualitatively correct in this regime. 
The simulation by Shiokawa et al. (2015) considers the encounter between a white dwarf and an intermediate-mass black hole ${ }^{20}$ with $M_{\mathrm{h}}=500 \mathrm{M}_{\odot}$ assuming an adiabatic evolution for the gas. Due to weak relativistic apsidal precession, this work finds that the stream intersects itself near the apocenter of its most bound part. This intersection radius is qualitatively similar to that in a large fraction of real TDEs, namely those with $\beta \approx 1$ and $M_{\mathrm{h}} \lesssim 2 \times 10^{6} \mathrm{M}_{\odot}$ (see Section 3.1.2). ${ }^{21}$ Around this location, the two colliding components are both thick, with widths $H_{1} \approx H_{2} \lesssim R_{\text {int }}=a_{\text {min }}$ (see Fig. 3) similar to the intersection radius. This is likely a consequence of the increased dynamical impact of the nozzle shock, which for low-mass black holes causes the stream to significantly expand before it first intersects itself (see Section 3.2). Interactions between these thick streams are therefore enhanced relative to the weaker collision that might be expected for thin streams, in a way that may compensate for the weak relativistic apsidal precession, as discussed in Section 3.1.2. As can be seen from the snapshots of Fig. 11, the resulting self-crossing shock initially induces mild changes in the gas trajectories associated with a slow expansion that is unable to unbind mass from the black hole. Early on, most of the dissipation still occurs at the nozzle shock, although this interaction weakens due to an expansion of the returning stream. The interactions resulting from the self-crossing shock (termed "outer shocks" in this work) are analysed in detail, and decomposed into a "forward" and "reverse" component that are initially close in space. At later times, the reverse one recedes closer to the black hole as mass accumulates near apocenter while the forward component remains around the same location. Several passages of the returning gas through this shock system progressively lead to a redistribution of the gas orbital elements accompanied by an overall decrease of its eccentricities. During this process, a significant fraction of the gas has its angular momentum diminished so that it reaches scales similar to the black hole event horizon that can dominate the early accretion, as we discuss more in detail in Section 3.5.2. The accretion flow remains globally eccentric for about ten dynamical times before settling into a more axisymmetric structure (i.e. $t_{\text {circ }} \approx 10 t_{\text {min }}$ ) that is thick with internal eccentricities of $e \approx 0.3$ on average, and extends out to a radius $R_{\mathrm{d}} \approx a_{\text {min }}$ similar to the semi-major axis of the most bound debris. As was also the case for the weak self-crossing shock captured by the above works (e.g. Bonnerot et al. 2016) considering bound stars with $\beta \approx 1$, this simulation suggests that circularization is slowed when relativistic apsidal precession is reduced. However, it is unclear whether these results obtained for an intermediate-mass black hole can be extrapolated to the more common situation of a solar-type star disrupted by a supermassive black hole. An im-

\footnotetext{
20 Numerical studies considering intermediate-mass black holes were carried out earlier as well (Rosswog et al. 2008, 2009; Ramirez-Ruiz and Rosswog 2009; Guillochon et al. 2014). However, they do not run for long enough to capture the completion of disc formation, and we therefore do not present them in detail here.

21 For the parameters used by Shiokawa et al. (2015), the relativistic apsidal angle is the same as for the disruption of a solar-type star by a black hole of mass $M_{\mathrm{h}}=3 \times 10^{5} \mathrm{M}_{\odot}$ for $\beta=1$.
} 
portant source of uncertainty relates to the increased thickness of the debris, that may significantly modify the gas evolution compared to that involving a thin returning stream confined by self-gravity (see Section 2).

The above simulations aim at studying the global hydrodynamics of accretion flow formation in TDEs. Along with the works presented in Section 3.1, they constitute our current understanding of this complex process, that is to date still highly uncertain, although clearly different from the early prediction by Rees (1988). Despite the simplified initial conditions used, these numerical investigations allow us to gain insight into the astrophysical problem and identify the main remaining sources of uncertainty. If a powerful self-crossing shock takes place near pericenter that strongly modifies the trajectories of the colliding gas, there is convincing evidence that at least a fraction of the debris can promptly form an accretion flow with $t_{\text {circ }} \lesssim t_{\text {min }}$, whose properties may be qualitatively similar to those found in some simulations (e.g. Sadowski et al. 2016; Bonnerot and Lu 2019). However, the exact region of parameter space corresponding to this regime and the detailed gas evolution here are still not clearly identified. If the stream intersects itself around apocenter due to weak apsidal precession, some works above (Shiokawa et al. 2015; Bonnerot et al. 2016) suggest a much slower formation of the accretion flow. However, it remains to be understood how sensitive these results are to parameter choices made for computational tractability. Tidal disruption by intermediate-mass black holes likely produces streams of much larger relative width due to the enhanced importance of the nozzle shock, while eccentric stellar orbits can lead to unphysical initial interactions between streams (e.g. "head chasing tail" behavior) that are probably of greater importance for very weak stream intersections. For these reasons, important uncertainties still exist in this regime, with the possibility that the gas evolves in a realistic situation very differently from what current simulations predict. For example, it is possible that the debris forms a disc that largely fails to circularize by retaining eccentricities $e \approx e_{\min }$ for multiple orbital timescales. Overall, accretion flow formation remains a very open problem, in which the hydrodynamics is not yet qualitatively understood for a large fraction of the parameter space. Progress is likely to come from a better understanding of the early sources of dissipation discussed in Section 3.1 combined with improved global simulations more directly applicable to the astrophysically realistic situation.

Regarding the inclusion of additional physics, the influence of black hole spin on this process is also uncertain since it has so so far only been studied for bound stars (Hayasaki et al. 2016; Liptai et al. 2019). The impact of nodal precession at preventing a prompt self-crossing shock remains to be evaluated for a parabolic encounter that would in particular require to estimate the expansion induced by the nozzle shock in this situation (see Section 3.1.1). Another important effect relates to the radiative efficiency, that may increase as the gas distribution becomes more extended due to a decrease of its optical depth, as is explained in more detail in Section 3.4. The gas evolution would then deviate from that assuming complete adiabaticity, which may in particular cause the newly-formed disc to get thinner and more compact as 
predicted from isothermal runs (Hayasaki et al. 2013; Bonnerot et al. 2016; Hayasaki et al. 2016). Evaluating the exact level of radiative cooling requires to carry out radiation-hydrodynamics simulations of this process that has not been done so far. Despite the several uncertainties we mentioned, the results of current simulations have led to major improvements in our understanding of accretion flow formation. In the future, additional progress will come from greater computational resources and the development of innovative numerical schemes that would allow us to circumvent the computational limitations presented in Section 3.2.

\subsection{Shock radiative efficiency and resulting emission}

Before settling into an accretion disc, the numerical works presented in Section 3.3 show that the returning debris experiences a large amount of dissipation. The outcome of these shocks depends on their radiative efficiency, that is in turn determined by the optical depth of the surrounding matter. For a very optically thick gas, the photons are advected with the fluid element that created them. In this case, the interaction is radiatively inefficient and the hydrodynamics is close to completely adiabatic. In contrast, more optically thin matter allows photons to be transported away from their production site by diffusion, and to potentially leave the system entirely. The gas evolution may then significantly deviate from the adiabatic limit, and the escaping radiation can participate in the electromagnetic signal observed from TDEs, as discussed more in details in the Emission Mechanisms Chapter. We start by evaluating the radiative efficiency of the initial self-crossing shock and then focus on the later interactions that result in accretion flow formation.

As described in Section 3.1.2, the outcome of the self-crossing shock remains unclear since it depends on several effects whose relative importance has so far not been precisely estimated. As before, we treat first the idealized situation where the collision involves aligned stream components with the same thin width $H_{1}=H_{2} \ll R_{\text {int }}$, and then discuss possible deviations from these strong assumptions. In this regime, the shock-heating rate is obtained from $\dot{E}_{\mathrm{sc}}^{\max }=$ $\dot{M}_{\mathrm{fb}} \Delta \varepsilon_{\mathrm{sc}}^{\max }$ by multiplying the fallback rate at which the gas enters the collision by the specific energy dissipated. Using equation (15) then yields

$$
\dot{E}_{\mathrm{sc}}^{\max } \approx \frac{G M_{\mathrm{h}} \dot{M}_{\mathrm{fb}}}{R_{\mathrm{int}}} \approx 7 \times 10^{43} \operatorname{erg~s}^{-1}\left(\frac{\mathrm{R}_{\mathrm{int}}}{\mathrm{a}_{\mathrm{min}}}\right)^{-1}\left(\frac{\mathrm{M}_{\mathrm{h}}}{10^{6} \mathrm{M}_{\odot}}\right)^{-1 / 6}
$$

where the numerical value assumes a collision angle $\psi \approx \pi$ and an intersection radius $R_{\text {int }} \approx a_{\text {min }}$ that is valid for the typical parameters $M_{\mathrm{h}}=10^{6} \mathrm{M}_{\odot}$ and $\beta=1$, and we have set the fallback rate equal to its peak value, with $\dot{M}_{\mathrm{fb}}=\dot{M}_{\mathrm{p}}$ using equation (6). This substantial rate of energy dissipation is comparable to the peak optical/UV luminosities observed in typical TDEs, which led Piran et al. (2015) to first propose it as the primary power source 
for these flares. ${ }^{22}$ Photons are emitted by the shocked gas with the resulting radiation pressure driving the formation of an outflow that, for simplicity, we approximate as quasi-spherical (see left panel of Fig. 3) with a density profile $\rho_{\text {out }} \approx \dot{M}_{\mathrm{fb}} /\left(4 \pi R^{2} v_{\text {out }}\right)$ where $R$ denotes the distance from the intersection point. The diffusion and dynamical timescales given by $t_{\text {diff }} \approx R \tau_{\mathrm{s}} / c$ and $t_{\mathrm{dyn}}=R / v_{\text {out }}$ are equal at the trapping radius $R_{\mathrm{tr}}$ where the scattering optical depth is $\tau_{\mathrm{s}} \equiv \int_{R_{\mathrm{tr}}}^{\infty} \kappa_{\mathrm{s}} \rho_{\text {out }} \mathrm{d} R=c / v_{\text {out }}$, yielding $R_{\mathrm{tr}}=\dot{M}_{\mathrm{fb}} \kappa_{\mathrm{s}} /(4 \pi c)$, where $\kappa_{\mathrm{s}}=0.34 \mathrm{~cm}^{2} \mathrm{~g}^{-1}$ is the electron-scattering opacity. Radiation is coupled to the gas inside this radius and its energy is therefore advected outward at a rate $\dot{E}=4 \pi e_{\mathrm{r}} v_{\text {out }} R^{2} \propto R^{-2 / 3}$ where the last scaling uses the fact that the radiation energy density evolves adiabatically as $e_{\mathrm{r}} \propto \rho_{\text {out }}^{4 / 3} \propto R^{-8 / 3}$. This decreasing rate implies that photons lose energy as they are transported outward due to work done on the gas through radiation pressure. After being injected by shocks near the common width $H$ of the two colliding streams, at the luminosity given by equation (16), radiation energy is degraded in this way until photons reach the trapping radius. The emerging luminosity $L_{\mathrm{sc}}^{\max }$ is therefore reduced by a factor

$$
\frac{L_{\mathrm{sc}}^{\max }}{\dot{E}_{\mathrm{sc}}^{\max }} \approx\left(\frac{H}{R_{\mathrm{tr}}}\right)^{2 / 3} \approx 0.03\left(\frac{M_{\mathrm{h}}}{10^{6} \mathrm{M}_{\odot}}\right)^{1 / 3}\left(\frac{H}{10 \mathrm{R}_{\odot}}\right)^{2 / 3}
$$

where the numerical value assumes again $\dot{M}_{\mathrm{fb}}=\dot{M}_{\mathrm{p}}$ and uses a stream width $H \approx 10 \mathrm{R}_{\odot}$ that corresponds to an infalling stream confined by self-gravity (see Section 2) (Lu and Bonnerot 2019). In this regime, the self-crossing shock is therefore radiatively inefficient, a result in agreement with radiationhydrodynamics simulations of the collision assuming thin streams (Jiang et al. $2016 \mathrm{~b}$ ), and that justifies an assumption of adiabaticity for the gas evolution. For $M_{\mathrm{h}} \approx 10^{6} \mathrm{M}_{\odot}$, the resulting luminosity is $L_{\mathrm{sc}}^{\max } \approx 3 \times 10^{42} \mathrm{erg} \mathrm{s}^{-1}$, which seems too low to account for the brightest optical and UV emission detected from TDEs. If a significant fraction of the gas collides near pericenter with $R_{\text {int }} \approx R_{\mathrm{p}}$, as is common for $M_{\mathrm{h}} \gtrsim 10^{7} \mathrm{M}_{\odot}$, the heating rate of equation (16) could increase and result in a larger luminosity of $L_{\mathrm{sc}}^{\max } \gtrsim 10^{43} \mathrm{erg} \mathrm{s}^{-1}$. As discussed in Section 3.1.2, the two stream components involved in the selfcrossing shock may have significantly different widths, with $H_{2}>H_{1}$. In this case, we have seen that the specific energy dissipated is likely lower than that used in equation (16), and this would tend to decrease the emerging luminosity compared to $L_{\mathrm{sc}}^{\max }$. However, a thicker gas distribution accompanied by a significant deviation from spherical geometry for the shocked gas could instead result in a more radiatively efficient interaction, with lower adiabatic losses than is predicted in the above case of identical streams. Our current understanding of the hydrodynamics of a realistic self-crossing shock would have to be improved to go beyond these qualitative expectations.

\footnotetext{
22 Earlier on, Lodato (2012) also estimated the energy that must be radiated for the gas to completely circularize, proposing that this could be an observable power source without further development.
} 
Following the self-crossing shock, the gas undergoes additional interactions that may circularize the orbits to form an accretion flow. We now focus on the radiation emerging during this later stage, bearing in mind that it may not always be possible to distinguish it from the initial collision, especially if a significant fraction of the gas starts accumulating near the black hole as a result of the self-crossing shock alone. ${ }^{23}$ If these circularizing shocks can happen closer to the black hole, they are likely stronger than the initial collision. In the work by Bonnerot and $\mathrm{Lu}(2019)$ that adopts realistic astrophysical parameters, the corresponding heating rate reaches $\dot{E}_{\mathrm{sh}} \approx 10^{44} \mathrm{erg} \mathrm{s}^{-1}$. Notably, Piran et al. (2015) extrapolates a similar value from the simulations by Shiokawa et al. (2015) that considers an intermediate-mass black hole. As these interactions take place, we assume that the gas distribution has expanded to fill an approximately spherical region within a distance $R_{\mathrm{d}}$ from the black hole. If it contains about the mass of the original star, the optical depth of this gas may be estimated as $\tau_{\mathrm{s}} \approx \kappa_{\mathrm{s}} M_{\star} /\left(4 \pi R_{\mathrm{d}}^{2}\right)$. Comparing the diffusion timescale $t_{\mathrm{diff}} \approx R_{\mathrm{d}} \tau_{\mathrm{s}} / c$ to the dynamical time $t_{\mathrm{dyn}} \approx 2 \pi\left(G M_{\mathrm{h}} / R_{\mathrm{d}}^{3}\right)^{-1 / 2}$ then yields

$$
\frac{t_{\mathrm{diff}}}{t_{\mathrm{dyn}}} \approx 1.5\left(\frac{M_{\mathrm{h}}}{10^{6} \mathrm{M}_{\odot}}\right)^{-7 / 6}\left(\frac{R_{\mathrm{d}}}{a_{\mathrm{min}}}\right)^{-7 / 2},
$$

where the numerical value adopts a radius $R_{\mathrm{d}}=a_{\min }$ as motivated by the simulations described in Section 3.3. This simple estimate (Piran et al. 2015; Hayasaki et al. 2016) suggests that the circularizing shocks are radiatively efficient for $M_{\mathrm{h}} \gtrsim 10^{6} \mathrm{M}_{\odot}$. As a result, the emerging luminosity may therefore be similar to the heating rate with $L_{\mathrm{sh}} \approx \dot{E}_{\mathrm{sh}}$, which is likely larger than that originating from the self-crossing shock in most cases. This mechanism therefore represents a plausible explanation for the observed optical and UV luminosities, although this origin may be difficult to distinguish from that associated with gas accretion (Metzger and Stone 2016; Bonnerot and Lu 2019). As mentioned in Section 3.3, a significant fraction of the internal energy injected by shocks may be lost from the system as a result of this increased radiative efficiency. The accretion disc could therefore be initially thinner than expected from the numerical works assuming complete adiabaticity. Radiationhydrodynamics simulations of this process are required to precisely evaluate the emerging electromagnetic signal and the impact of radiative cooling on the gas evolution.

\subsection{Implications for the nascent accretion flow}

The outcome of the complex, three-dimensional hydrodynamical evolution described in Section 3.3 represents the initial conditions from which accretion onto the black hole will proceed. A detailed analysis of accretion processes

\footnotetext{
23 This situation is for example expected if early interactions take place near pericenter either as a result of strong relativistic apsidal precession or due to gas deflection induced by a fast expansion at the nozzle shock (see Section 3.1.2).
} 
is provided in the Accretion Disc Chapter and we only emphasize here the properties that can be extracted from the gas evolution at play during disc formation. The main reason for doing so is that most simulations of disc accretion in TDEs are, so far, initialized from an already formed disc, mostly for computational reasons that prevent us from self-consistently following the prior disk assembly process. It is therefore not guaranteed that these frequently used initial conditions possess all the properties described here, and this should be kept in mind by the reader when going through the next chapter.

\subsubsection{Slow gas inflow: no $t^{-5 / 3}$ decay of the mass accretion rate?}

As we have already highlighted, the current picture of disc formation is drastically different from the pioneering work by Rees (1988), that assumes the fast formation of a compact accretion disc on scales similar to $R_{\mathrm{p}}$. Instead, numerical simulations find that disc formation can be a slow process with most of the gas settling at distances $R_{\mathrm{d}} \gg R_{\mathrm{p}}$ larger than initially thought (Shiokawa et al. 2015; Sadowski et al. 2016; Bonnerot and Lu 2019). Assuming a standard $\alpha$-disc, the viscous timescale $t_{\text {visc }}$ at this location is given by the ratio (Frank et al. 2002)

$$
\frac{t_{\mathrm{visc}}}{t_{\min }} \approx \frac{1}{\alpha}\left(\frac{R_{\mathrm{d}}}{a_{\min }}\right)^{3 / 2}\left(\frac{H}{R}\right)^{-2}
$$

from which we see that $t_{\text {visc }} / t_{\min } \approx 10$ if $R_{\mathrm{d}} \approx a_{\min }$. Here, the viscous parameter and the aspect ratio have been given reasonable values of $\alpha \approx 0.1$ and $H / R \approx 1$. This implies that accretion through the disc may be slow, such that a large amount of mass is accumulated at large radii with no significant, time-averaged inflow motion even long after the disc has settled into a quasi-steady state. The early model by Rees (1988) predicted that the mass accretion rate follows the same decay law (of $t^{-5 / 3}$ ) as the fallback rate, based on the assumptions of rapid disc formation and efficient viscous processing of the freshly returning matter. According to the above analysis, these conditions may not be realized, suggesting a different evolution of the inflow rate through the disc.

\subsubsection{Ballistic accretion and advection: solutions to the "inverse energy crisis"?}

The total energy radiated in the optical, near-UV, and soft X-ray from most observed TDE candidates is almost always $\lesssim 10^{51} \mathrm{erg}$, a value about three orders of magnitude lower than the rest mass energy of a solar-type star. Additionally, the energy loss necessary for complete circularization is $M_{\star} \Delta \varepsilon_{\text {circ }} \approx$ $10^{52} \mathrm{erg}$ according to equation (9), which also significantly exceeds the observed values. This results in an "inverse energy crisis" where less energy is detected than one would have expected (Piran et al. 2015; Stone and Metzger 2016; Lu and Kumar 2018). We focus below on proposed solutions for this 
puzzle that involve the hydrodynamics of the accretion flow, but also refer to other possibilities for completeness.

The low energetics of observed events can be explained if most of the gas is ballistically accreted onto the black hole, carrying its orbital energy below the event horizon without any dissipation. As mentioned in Section 3.3, ballistic accretion has been found in several simulations (Shiokawa et al. 2015; Sadowski et al. 2016; Bonnerot and Lu 2019) of the disc formation process, which find that a significant fraction of the debris gets accreted with significant eccentricities. This process is caused by angular momentum redistribution happening during the circularizing shocks and could reduce the radiative efficiency of the flow. A more extreme scenario proposed by Svirski et al. (2017) involves an highly eccentric disc with $e \approx e_{\min }$ that is a possible outcome of the disc formation process but has not been found in current simulations. In this situation, magnetic stresses are more efficient at transporting angular momentum near apocenter that acts to progressively reduce the pericenter of the debris. These authors propose that this mechanism can keep operating until all the gas enters the event horizon of the black hole, thereby strongly suppressing the radiation produced by either circularization or accretion. However, another effect of reducing the gas pericenter that close to the gravitational radius is to strongly enhance relativistic apsidal precession. As argued by Bonnerot et al. (2017), the net impact could therefore be to enhance interactions between different parts of this disc, promoting dissipation. A related possibility is that significant dissipation actually occurs inside the forming disc but with a subsequent advection of the injected internal energy, as proposed by Begelman (1979) and Abramowicz et al. (1996).

Although alleviating the tension, it is not clear whether hydrodynamical models completely solve it and another perhaps more natural solution relies on the fact that the disc emits in the extreme ultraviolet (EUV) part of the spectrum that is not observable (Lu and Kumar 2018). This solution has gained recent support observationally through the detection of infrared dust echoes (Jiang et al. 2016a) and Bowen resonance lines (Blagorodnova et al. 2019) from TDE candidates that are both known to be triggered by extremeultraviolet radiation. Indeed, simple models for the infrared dust echoes observed in the TDE flares PTF-09ge and PTF-09axc infer EUV luminosities $\sim 10^{44-45} \mathrm{erg} \mathrm{s}^{-1}$ (van Velzen et al. 2016). The total EUV energy release from these flares is uncertain, but in both cases could be $\sim 10^{52} \mathrm{erg} \mathrm{s}^{-1}$, which would solve the inverse energy crisis if one assumes disruption of lower main sequence stars with masses $M_{\star} \lesssim 0.3 M_{\odot}$. While this could be a promising solution, we caution that the modeling of these dust echoes involves many assumptions on the interstellar dust composition and geometry.

\subsubsection{Initial source of disc effective viscosity}

In most accreting systems, angular momentum transport is driven by magnetic stresses resulting from the magneto-rotational instability (MRI) (Balbus and Hawley 1991) that are often parametrized in steady state as an effec- 
tive viscosity. However, it is not obvious that the early phase of accretion in TDEs is produced by the same physical process. This possibility has been put forward by the magneto-hydrodynamical simulations of Sadowski et al. (2016), who found that even after the MRI has reached saturation, angular momentum transport is still dominated by purely hydrodynamical turbulence, which they attribute to either convection or the perturbation created by the matter originally ejected from the self-crossing shock and continuously joining the newly-formed disc. ${ }^{24}$ Similarly, the simulation by Bonnerot and Lu (2019) finds that the forming disc contains spiral shocks excited by later-arriving matter that continuously strikes its outer edge. Angular momentum is transported outward at these locations, which produces a slow inflow through the mid-plane. Nealon et al. (2018) also proposed that the gas accretion at early times could be produced by angular momentum transport associated to the Papaloizou-Pringle instability (Papaloizou and Pringle 1984), whose growth timescale was evaluated to be shorter than that of the MRI owing to the low initial disc magnetic field inherited from the disrupted star. Finally, the development of MRI may differ in eccentric discs forming from debris that fails to fully circularize (Chan et al. 2018).

\section{Summary and conclusion}

In this chapter, we have presented the current understanding of stream evolution prior to pericenter return (Section 2) and the formation of an accretion flow around the black hole from the bound part of this gas (Section 3).

The first phase is well-understood: different stream elements move on ballistic orbits, while their transverse motion is usually at first set by the gas self-gravity (Section 2.1). Several authors have also investigated the influence of various additional physical effects on the stream evolution (Section 2.2). Gravitational fragmentation inside this gas distribution can lead to the formation of clumps, while the confinement by self-gravity can be overcome by either magnetic pressure or through heating associated with hydrogen recombination. It is also possible that the debris experiences interactions with the surrounding medium that can enhance its mixing with this ambient gas. Nevertheless, this additional physics does not appear to drastically change the basic picture of how the stream evolves before its bound part comes back to pericenter.

Our understanding of the second phase of accretion disc formation is less robust. The initial dissipation (Section 3.1) is in most cases dominated by a self-crossing shock whose characteristics are specified by the combined effect of relativistic apsidal precession, expansion from pericenter due to the nozzle shock, and nodal precession produced by the black hole spin. When apsidal precession dominates, local simulations initialized with thin identical stream components find that the collision results in a quasi-spherical expansion of the

\footnotetext{
24 We note, however, that over much of the computational domain of Sadowski et al. (2016), the magnetorotational instability is marginally or under-resolved.
} 
gas. However, this evolution may significantly change if the nozzle shock imparts a large width difference to the streams or if nodal precession delays the first intersection and causes the collision to be misaligned. Studying the later hydrodynamics requires global simulations that are very numerically challenging owing to the large dynamic range involved, and have therefore only been carried out so far for simplified initial conditions (Section 3.2). These works find that the debris experiences additional interactions, causing it to progressively move to more circular orbits until eventually settling into an accretion flow (Section 3.3). This process appears to complete on a shorter timescale for more relativistic stellar pericenters, due to an increased strength of the initial self-crossing shock. If the gas evolves adiabatically, the final outcome is a thick and extended distribution that typically retains significant internal eccentricities. However, due to the unrealistic setup used in these numerical studies, the precise hydrodynamics at play during accretion flow formation is not yet established and it therefore cannot be excluded that its outcome is different from what current simulations predict. For example, encounters with weak apsidal precession may lead to a gas distribution that completely fails to circularize and remain instead highly eccentric. As the disc assembles, the radiation produced during circularizing shocks may leave the system to participate to the emerging luminosity from TDEs, especially in the optical/UV bands (Section 3.4). The outcome of the disc formation process represents the initial state for the subsequent accretion onto the black hole and the above works suggest that this later phase consists in a slow inflow of gas induced by effective viscosity with a possibly important contribution from ballistic accretion (Section 3.5).

Building on the progress made so far, improvements in our understanding of the complex process of accretion flow formation will come in the future from a combination of systematic studies of the different mechanisms involved and global simulations of the entire hydrodynamics applicable to an astrophysically realistic situation. Additional physics missing from most current works such as radiative diffusion, black hole spin and magnetic fields should also be incorporated in these investigations since their influence can significantly affect the gas evolution. Some of these advancements are already being undertaken and will lead to major progress in the theoretical understanding of TDEs.

Acknowledgements We gratefully acknowledge conversations with, and detailed comments from T. Piran, as well as his edits on an earlier version of this manuscript. We are also grateful to E.M. Rossi for insightful discussions during the writing of this chapter. The research of $\mathrm{CB}$ was funded by the Gordon and Betty Moore Foundation through Grant GBMF5076. NCS was supported by the NASA Astrophysics Theory Research program (grant NNX17AK43G; PI B. Metzger), and from the Israel Science Foundation (Individual Research Grant 2565/19).

\section{References}

M.A. Abramowicz, X.M. Chen, M. Granath, J.P. Lasota, advection-dominated accretion flows around Kerr Black holes. Astrophys. J. 10, 762-773 (1996). doi:10.1086/178004 
P. Amaro-Seoane, M.C. Miller, G.F. Kennedy, Tidal disruptions of separated binaries in galactic nuclei. Mon. Not. R. Astron. Soc. 425(4), 2401-2406 (2012). doi:10.1111/j.13652966.2012.21162.x

S. Ayal, M. Livio, T. Piran, Tidal Disruption of a Solar-Type Star by a Supermassive Black Hole. Astrophys. J. 545, 772-780 (2000). doi:10.1086/317835

S.A. Balbus, J.F. Hawley, A powerful local shear instability in weakly magnetized disks. I - Linear analysis. Astrophys. J. 376(9), 214 (1991). ISBN 9788578110796. doi:10.1086/170270

M.C. Begelman, Can a spherically accreting black hole radiate very near the Eddington limit. Mon. Not. R. Astron. Soc. 187(2), 237-251 (1979). doi:10.1093/mnras/187.2.237

N. Blagorodnova, S.B. Cenko, S.R. Kulkarni, I. Arcavi, J.S. Bloom, G. Duggan, A.V. Filippenko, C. Fremling, A. Horesh, G. Hosseinzadeh, E. Karamehmetoglu, A. Levan, F.J. Masci, P.E. Nugent, D.R. Pasham, S. Veilleux, R. Walters, L. Yan, W. Zheng, The Broad Absorption Line Tidal Disruption Event iPTF15af: Optical and Ultraviolet Evolution. Astrophys. J. 873(1), 92 (2019). doi:10.3847/1538-4357/ab04b0

L. Blanchet, Gravitational radiation from post-newtonian sources and inspiralling compact binaries. Living Rev. Relativ. 17(2014) (2014). doi:10.12942/lrr-2014-2

C. Bonnerot, W. Lu, Simulating realistic disc formation in tidal disruption events. ArXiv e-prints 19(June), 1-19 (2019)

C. Bonnerot, E.M. Rossi, Streams collision as possible precursor of double tidal disruption events. Mon. Not. R. Astron. Soc. 484(1), 1301-1316 (2019). doi:10.1093/mnras/stz062

C. Bonnerot, E.M. Rossi, G. Lodato, Bad prospects for the detection of giant stars' tidal disruption: Effect of the ambient medium on bound debris. Mon. Not. R. Astron. Soc. 458(3), 3324-3330 (2016). doi:10.1093/mnras/stw486

C. Bonnerot, E.M. Rossi, G. Lodato, Long-term stream evolution in tidal disruption events. Mon. Not. R. Astron. Soc. 15(August), 1-15 (2017). doi:10.1093/mnras/stw2547

C. Bonnerot, E.M. Rossi, G. Lodato, D.J. Price, Disc formation from tidal disruptions of stars on eccentric orbits by Schwarzschild black holes. Mon. Not. R. Astron. Soc. 455(2), 2253-2266 (2016). doi:10.1093/mnras/stv2411

C. Bonnerot, D.J. Price, G. Lodato, E.M. Rossi, Magnetic field evolution in tidal disruption events. Mon. Not. R. Astron. Soc. 469(4), 4879-4888 (2017). doi:10.1093/mnras/stx1210

M. Brassart, J.P. Luminet, Shock waves in tidally compressed stars by massive black holes. Astron. \& Astrophys. 481, 259 (2008). doi:10.1051/0004-6361:20078264

J.K. Cannizzo, H.M. Lee, J. Goodman, The disk accretion of a tidally disrupted star onto a massive black hole. Astrophys. J. 351, 38 (1990). doi:10.1086/168442

B. Carter, J.P. Luminet, Pancake detonation of stars by black holes in galactic nuclei. Nature 296(5854), 211-214 (1982). doi:10.1038/296211a0

C.-H. Chan, J.H. Krolik, T. Piran, Magnetorotational Instability in Eccentric Disks. Astrophys. J. 856(1), 12 (2018). doi:10.3847/1538-4357/aab15c

C.-H. Chan, T. Piran, J.H. Krolik, D. Saban, Tidal disruption events in active galactic nuclei. ArXiv e-prints (2019)

R.M. Cheng, T. Bogdanović, Tidal disruption of a star in the Schwarzschild spacetime: Relativistic effects in the return rate of debris. Phys. Rev. D 90(6), 064020 (2014). doi:10.1103/PhysRevD.90.064020

E.R. Coughlin, C. Nixon, Variability in Tidal Disruption Events: Gravitationally Unstable Streams. Astrophys. J. Lett. 808, 11 (2015). doi:10.1088/2041-8205/808/1/L11

E.R. Coughlin, C. Nixon, M.C. Begelman, P.J. Armitage, On the structure of tidallydisrupted stellar debris streams. Mon. Not. R. Astron. Soc. 17(March), 1-17 (2016a). doi:10.1093/mnras/stw770

E.R. Coughlin, C. Nixon, M.C. Begelman, P.J. Armitage, D.J. Price, Post-periapsis pancakes: Sustenance for self-gravity in tidal disruption events. Mon. Not. R. Astron. Soc. 455(4), 3612-3627 (2016b). doi:10.1093/mnras/stv2511

L. Dai, A. Escala, P. Coppi, the Impact of Bound Stellar Orbits and General Relativity on the Temporal Behavior of Tidal Disruption Flares. Astrophys. J. 775(1), 9 (2013). doi:10.1088/2041-8205/775/1/L9

L. Dai, J.C. McKinney, M.C. Miller, Soft X-Ray Temperature Tidal Disruption Events From Stars on Deep Plunging Orbits. Astrophys. J. 812(2), 39 (2015). doi:10.1088/2041- 
8205/812/2/L39

S. Darbha, E.R. Coughlin, D. Kasen, C. Nixon, Ultra-deep tidal disruption events: prompt self-intersections and observables. Mon. Not. R. Astron. Soc. (2019). doi:10.1093/mnras/stz1923

C. Evans, P. Laguna, M. Eracleous, Ultra-Close Encounters of Stars With Massive Black Holes: Tidal Disruption Events With Prompt Hyperaccretion. Astrophys. J. 805(2), 19 (2015). doi:10.1088/2041-8205/805/2/L19

G. Faye, L. Blanchet, A. Buonanno, Higher-order spin effects in the dynamics of compact binaries. I. Equations of motion. Phys. Rev. D 74(10), 104033 (2006). doi:10.1103/PhysRevD.74.104033

J. Frank, A. King, D.s. Raine, Accretion Power in Astrophysics: Third Edition (Cambridge University Press, Cambridge, 2002), p. 398

E. Girma, J. Guillochon, The Galactic Distribution of Fragments Formed from Tidally Disrupted Stars. ArXiv e-prints, 1707-03421 (2018)

J. Guillochon, M. McCourt, Simulations of Magnetic Fields in Tidally Disrupted Stars. Astrophys. J. 834(2), 19 (2017). doi:10.3847/2041-8213/834/2/L19

J. Guillochon, E. Ramirez-Ruiz, A DARK YEAR FOR TIDAL DISRUPTION EVENTS. Astrophys. J. 809, 166 (2015). doi:10.1088/0004-637X/809/2/166

J. Guillochon, H. Manukian, E. Ramirez-Ruiz, PS1-10jh: THE DISRUPTION OF A MAINSEQUENCE STAR OF NEAR-SOLAR COMPOSITION. Astrophys. J. 783(1), 23 (2014). doi:10.1088/0004-637X/783/1/23

R. Haas, R.V. Shcherbakov, T. Bode, P. Laguna, Tidal Disruptions of White Dwarfs From Ultra-Close Encounters With Intermediate-Mass Spinning Black Holes. Astrophys. J. 749(2), 117 (2012). doi:10.1088/0004-637X/749/2/117

K. Hayasaki, N. Stone, A. Loeb, Finite, intense accretion bursts from tidal disruption of stars on bound orbits. Mon. Not. R. Astron. Soc. 434(2), 909-924 (2013). doi:10.1093/mnras/stt871

K. Hayasaki, N. Stone, A. Loeb, Circularization of tidally disrupted stars around spinning supermassive black holes. Mon. Not. R. Astron. Soc. 461(4), 3760-3780 (2016) doi:10.1093/mnras/stw1387

M.s. Hobson, G.s. Efstathiou, A.s. Lasenby, General Relativity: An Introduction for Physicists (Cambridge University Press, Cambridge, 2006), p. 590

N. Jiang, L. Dou, T. Wang, C. Yang, J. Lyu, H. Zhou, THE WISE DETECTION OF AN INFRARED ECHO IN TIDAL DISRUPTION EVENT ASASSN-14li. Astrophys. J. 828(1), 14 (2016a). doi:10.3847/2041-8205/828/1/L14

Y.-F. Jiang, J. Guillochon, A. Loeb, Prompt Radiation and Mass Outflows From the StreamStream Collisions of Tidal Disruption Events. Astrophys. J. 830(2), 125 (2016b). doi:10.3847/0004-637X/830/2/125

V. Karas, L. Šubr, Enhanced activity of massive black holes by stellar capture assisted by a self-gravitating accretion disc. Astron. ES Astrophys. 19, 11-19 (2007). doi:10.1051/00046361

D. Kasen, E. Ramirez-Ruiz, Optical Transients From the Unbound Debris of Tidal Disruption. Astrophys. J. 714(1), 155-162 (2010). doi:10.1088/0004-637X/714/1/155

A. Kathirgamaraju, R.B. Duran, D. Giannios, TDE fallback cut-off due to a pre-existing accretion disc. ArXiv e-prints (2017)

L.Z. Kelley, A. Tchekhovskoy, R. Narayan, Tidal disruption and magnetic flux capture: Powering a jet from a quiescent black hole. Mon. Not. R. Astron. Soc. 445(4), 39193938 (2014). doi:10.1093/mnras/stu2041

S.S. Kim, M. Park, H.M. Lee, The StreamStream Collision after the Tidal Disruption of a Star around a Massive Black Hole. Astrophys. J. 519(2), 647-657 (1999). doi:10.1086/307394

C.S. Kochanek, The aftermath of tidal disruption: The dynamics of thin gas streams. Astrophys. J. 422, 508 (1994). doi:10.1086/173745

D. Liptai, D.J. Price, I. Mandel, G. Lodato, Disc formation from tidal disruption of stars on eccentric orbits by Kerr black holes using GRSPH. arXiv e-prints, 1910-10154 (2019)

G. Lodato, Challenges in the modeling of tidal disruption events lightcurves. EPJ Web Conf. 39, 01001 (2012). doi:10.1051/epjconf/20123901001

G. Lodato, D.J. Price, On the diffusive propagation of warps in thin accretion discs. Mon. 
Not. R. Astron. Soc. 405(2), 1212-1226 (2010). doi:10.1111/j.1365-2966.2010.16526.x

W. Lu, C. Bonnerot, Self-intersection of the Fallback Stream in Tidal Disruption Events. ArXiv e-prints 000(April) (2019)

W. Lu, P. Kumar, On the Missing Energy Puzzle of Tidal Disruption Events. Astrophys. J. 865(2), 128 (2018). doi:10.3847/1538-4357/aad54a

M. MacLeod, J. Goldstein, E. Ramirez-Ruiz, J. Guillochon, J. Samsing, Illuminating Massive Black Holes With White Dwarfs: Orbital Dynamics and High Energy Transients from Tidal Interactions. Astrophys. J. 794(1), 9 (2014). doi:10.1088/0004-637X/794/1/9

D. Merritt, Dynamics and Evolution of Galactic Nuclei (Princeton University Press, Princeton, 2013). ISBN 9780691158600

B.D. Metzger, N.C. Stone, A bright year for tidal disruptions. Mon. Not. R. Astron. Soc. 461(1), 948-966 (2016). doi:10.1093/mnras/stw1394

M.C. Miller, Disk Winds As an Explanation for Slowly Evolving Temperatures in Tidal Disruption Events. Astrophys. J. 805(1), 83 (2015). doi:10.1088/0004-637X/805/1/83

R. Nealon, D.J. Price, C. Bonnerot, G. Lodato, On the Papaloizou-Pringle instability in tidal disruption events. Mon. Not. R. Astron. Soc. 474(2), 1737-1745 (2018). doi:10.1093/mnras/stx2871

B. Paczyńsky, P.s.J. Wiita, Thick accretion disks and supercritical luminosities. Astron. EG Astrophys. 88, 23-31 (1980)

J.C.B. Papaloizou, J.E. Pringle, The dynamical stability of differentially rotating discs with constant specific angular momentum. Mon. Not. R. Astron. Soc. 208(4), 721-750 (1984). ISBN 9781479978007. doi:10.1093/mnras/208.4.721

T. Piran, G. Svirski, J. Krolik, R.M. Cheng, H. Shiokawa, Disk Formation Versus Disk AccretionWhat Powers Tidal Disruption Events? Astrophys. J. 806(2), 164 (2015). doi:10.1088/0004-637X/806/2/164

E. Ramirez-Ruiz, S. Rosswog, THE STAR INGESTING LUMINOSITY OF INTERMEDIATE-MASS BLACK HOLES IN GLOBULAR CLUSTERS. Astrophys. J. 697(2), 77-80 (2009). doi:10.1088/0004-637X/697/2/L77

M.J. Rees, Tidal disruption of stars by black holes of 106108 solar masses in nearby galaxies. Nature 333(6173), 523-528 (1988). ISBN 0028-0836. doi:10.1038/333523a0

S. Rosswog, E. Ramirez-Ruiz, W.R. Hix, Tidal Disruption and Ignition of White Dwarfs By Moderately Massive Black Holes. Astrophys. J. 695(1), 404-419 (2009). doi:10.1088/0004-637X/695/1/404

S. Rosswog, E. Ramirez-Ruiz, W.R. Hix, Atypical Thermonuclear Supernovae from Tidally Crushed White Dwarfs. Astrophys. J. 679(2), 1385-1389 (2008). doi:10.1086/528738

A. Sadowski, E. Tejeda, E. Gafton, S. Rosswog, D. Abarca, Magnetohydrodynamical simulations of a deep tidal disruption in general relativity. Mon. Not. R. Astron. Soc. 458(4), 4250-4268 (2016). doi:10.1093/mnras/stw589

N.I. Shakura, R.a. Sunyaev, Black holes in binary systems. Observational appearance. Astron. \& Astrophys. 24, 337-355 (1973). ISBN 9810200773. doi:10.1086/170270

H. Shiokawa, J.H. Krolik, R.M. Cheng, T. Piran, S.C. Noble, General Relativistic Hydrodynamic Simulation of Accretion Flow From a Stellar Tidal Disruption. Astrophys. J. 804(2), 85 (2015). doi:10.1088/0004-637X/804/2/85

E. Steinberg, E.R. Coughlin, N.C. Stone, B.D. Metzger, Thawing the frozen-in approximation: implications for self-gravity in deeply plunging tidal disruption events. Mon. Not. R. Astron. Soc. Lett. 485(1), 146-150 (2019). doi:10.1093/mnrasl/slz048

N.C. Stone, B.D. Metzger, Rates of stellar tidal disruption as probes of the supermassive black hole mass function. Mon. Not. R. Astron. Soc. 455(1), 859-883 (2016). doi:10.1093/mnras/stv2281

N. Stone, A. Loeb, Tidal disruption flares of stars from moderately recoiled black holes. Mon. Not. R. Astron. Soc. 422(3), 1933-1947 (2012). doi:10.1111/j.1365-2966.2012.20577.x

N. Stone, R. Sari, A. Loeb, Consequences of strong compression in tidal disruption events. Mon. Not. R. Astron. Soc. 435(3), 1809-1824 (2013). doi:10.1093/mnras/stt1270

G. Svirski, T. Piran, J. Krolik, Elliptical accretion and inefficient highly eccentric accretion and the low luminosity of stellar tidal disruption events. ArXiv e-prints (2017)

E. Tejeda, S. Rosswog, An accurate Newtonian description of particle motion around a Schwarzschild black hole. Mon. Not. R. Astron. Soc. 433(3), 1930-1940 (2013). doi:10.1093/mnras/stt853 
S. van Velzen, A.J. Mendez, J.H. Krolik, V. Gorjian, Discovery of Transient Infrared Emission From Dust Heated By Stellar Tidal Disruption Flares. Astrophys. J. 829(1), 19 (2016). doi:10.3847/0004-637X/829/1/19

C. Wegg, J.N. Bode, Multiple Tidal Disruptions As an Indicator of Binary Supermassive Black Hole Systems. Astrophys. J. 738(1), 8 (2011). doi:10.1088/2041-8205/738/1/L8

H.N. Wernke, A.-M. Madigan, The Effect of General Relativistic Precession on Tidal Disruption Events from Eccentric Nuclear Disks. ArXiv e-prints (2019) 